\title{
Decomposition driven interface evolution for layers of binary mixtures: II. Influence of convective transport on linear stability
}

\author{
Santiago Madrugat \\ Universidad Politécnica de Madrid, ETSI Aeronáuticos, \\ Plaza Cardenal Cisneros 3, 28040 Madrid, Spain \\ Uwe Thield \\ School of Mathematics, Loughborough University, \\ Loughborough, Leicestershire, LE11 3TU, UK
}

\begin{abstract}
We study the linear stability with respect to lateral perturbations of free surface films of polymer mixtures on solid substrates. The study focuses on the stability properties of the stratified and homogeneous steady film states studied in Part I [U. Thiele, S. Madruga and L. Frastia, Phys. Fluids 19, 122106 (2007)]. To this aim, the linearized bulk equations and boundary equations are solved using continuation techniques for several different cases of energetic bias at the surfaces, corresponding to linear and quadratic solutal Marangoni effects. For purely diffusive transport, an increase in film thickness either exponentially decreases the lateral instability or entirely stabilizes the film. Including convective transport leads to a further destabilization as compared to the purely diffusive case. In some cases the inclusion of convective transport and the related widening of the range of available film configurations (it is then able to change its surface profile) change the stability behavior qualitatively. We furthermore present results regarding the dependence of the instability on several other parameters, namely, the Reynolds number, the Surface tension number and the ratio of the typical velocities of convective and diffusive transport.
\end{abstract}




\section{INTRODUCTION}

The main stages of structure formation and dewetting in thin films of simple liquids are generally well understood $\underline{\underline{1}} \underline{\underline{6}}$. Films of polymers rupture forming holes, and evolve towards a network of liquid rims that may decay subsequently into small droplets ${ }^{7} \cdot \underline{8}$. Moreover, using physically/chemically heterogeneous substrates it is possible to create ordered soft matter films ${ }^{-13}$. For single component thin films there are two main pathways of dewetting: spinodal surface instability and heterogeneous nucleation, both driven by effective molecular interactions between the substrate and the film surface like, e.g., van der Waals forces. Both mechanisms may result in the rupture of the film leading to various patterns and have been studied extensively in numerical and experimental works. $\underline{\underline{14}} \underline{\underline{20}}$

The dewetting of thin films composed of binary mixtures is more involved. Experiments on mixtures have reported dewetting mechanisms that differ from the ones for simple liquids. In particular, Yerushalmi-Rozen et $a l^{21}$ discuss the phase-separation induced dewetting of a polymer blend consisting of deuterated oligomeric styrene and oligomeric ethylene-propylene. They assume that first there occurs a separation of the two components of the blend. The phase separation is followed by a dewetting process that is characterized by the formation of holes at a dewetting front moving inwards from the sample edge. This route of dewetting differs from the classical ones for single component films by its short induction time (at comparable film thickness), the properties of the front, and its morphological characteristics.

The evolving gradients of concentration of the two components of the binary mixture along the film surface originate surface tension gradients that are responsible for an additional flow in the vicinity of the decomposition/dewetting front. It is thought that this promotes the acceleration of the formation of holes. However, no detailed theoretical description is available at present. The relevance of interfacial phenomena in binary mixtures has as well been reported in spin-cast polymer blend films, where interfacial instabilities lead to a horizontal phase separation 22 .

Another element relevant to the dynamics of films of binary mixtures is the energetical influence of the surfaces. In particular, an energetically biased surface is rapidly enriched in the preferred component and may become the 'source' of so-called spinodal decomposition waves $23-25$. Jones et al $\stackrel{24}{\underline{4}}$, for instance, study the spinodal decomposition of critical mixtures of poly(ethylene-propylene) and its per-deuterated variant in presence of a substrate with 
a preferential attraction for the poly(ethylene-propylene). They find that that composition waves origin at the surfaces and propagate into the bulk. Their wave-vectors are oriented normal to the surface.

The interplay between phase separation, surface tension gradients, and surface properties make the dynamics of films of mixtures extremely rich and its theoretical description challenging. The dynamics of a fluid binary mixture is often described by the so called model-H, which couples transport of the mass of one component (convective Cahn-Hilliard equation) and momentum (Navier-Stokes-Korteweg equations)26,27. It has been extensively studied for various isothermal and non-isothermal settings $27-34$. None, however, involves beside the diffuse internal interface a free liquid-gas interface, i.e., a free surface. This implies that existing theories can not be readily applied to thin film experiments with polymer blends whose evolution is governed by the driving influence of the dynamics of and at the free surface.

In an alternative approach Clarke ${ }^{35,36}$ constructs and investigates a simple thin film model for the coupled evolution of film thickness profile and mean concentration. It is, however, based on the assumption that the films show no vertical concentration profile. This implies that it can not be applied to the case of the lateral stability of layered films we are interested in here.

The present series of works aims at the development and application of a model- $\mathrm{H}$ for systems involving free surfaces. Note that the incorporation of hydrodynamic flow is necessary even in the case of extremely slow creeping flow. Otherwise no evolving surface deflections can be described. The first part (Ref. 37) discusses the inclusion of free evolving surfaces into model-H and determines basic stratified film states for various types of energetic bias at the surfaces. In particular, the framework of phenomenological non-equilibrium thermodynamics is used to derive a generalized model-H coupling transport equations for momentum, density and entropy that is then simplified for an isothermal setting, vanishing interface viscosity and simplified internal energies. A comparison with literature results and a variational derivation of the static limiting case clarify the issue of defining pressure and chemical potential. Furthermore, boundary conditions at the solid substrate and the free interface are introduced. After non-dimensionalisation it is shown that the dimensionless numbers entering the boundary conditions for the Cahn-Hilliard and the Korteweg-NavierStokes equations are closely related and can not by any means be chosen independently of 
each other. Physically this means that the energetic bias with respect to decomposition at a free surface is intrinsically coupled to a solutal Marangoni effect. Neither of the two effects can be considered independently of the other one. Ref. 37 continues with an analysis of steady base state solutions for laterally homogeneous films of decomposing mixtures. A plethora of stratified solutions is determined and ordered for various types of energetic bias at the surfaces using continuation techniques and symmetry arguments.

This present second paper presents the analysis of the lateral stability of the laterally homogeneous steady films states obtained in Ref. 37, i.e., it determines the stability of homogeneous and layered films with respect to modulations in thickness and/or composition along the substrate. A similar approach (however, with other boundary conditions than used here) was recently employed to obtain instability thresholds for long-wave instabilities 34 . The paper is organized as follows. In Section II we review the non-dimensional model-H including appropriate boundary conditions at the solid substrate and the free film surface. In Section III equations are linearized for base states corresponding to homogeneous and stratified films. We as well discuss a realistic range of parameters for polymer blends to be used in the calculations. The stability results for homogeneous films are presented in Section IV for neutral and energetically biased surfaces. The stability of stratified films for neutral and biased surfaces is presented in Section V. Finally, conclusions are given in Section VI.

\section{DIMENSIONLESS MODEL}

We consider a film of a binary mixture on a horizontal homogeneous solid substrate. The film has a free surface that may evolve in time. The system is two-dimensional and infinitely extended in the horizontal direction. The origin of the Cartesian frame is fixed to the substrate. The dimensionless governing equations expressing the balance of mass and momentum, and the continuity equations are re-derived and discussed in Ref. 37. They read

$$
\begin{gathered}
\operatorname{Ps}_{\mathrm{S}}\left[\frac{\partial \mathbf{v}}{\partial t}+\mathbf{v} \cdot \nabla \mathbf{v}\right]=-\nabla \cdot\left\{(\nabla c)(\nabla c)+p_{\mathrm{eff}} \underline{\mathbf{I}}\right\}+\frac{\mathrm{PS}}{\mathrm{Re}} \Delta \mathbf{v}, \\
\partial_{t} c+\mathbf{v} \cdot \nabla c=-\nabla \cdot\left\{\nabla\left[\Delta c-\partial_{c} f(c)\right]\right\}
\end{gathered}
$$

and

$$
\nabla \cdot \mathbf{v}=0
$$


respectively, with the operators $\nabla=\left(\partial_{x}, \partial_{z}\right), \Delta=\left(\partial_{x}^{2}+\partial_{z}^{2}\right)$, and the velocity field $\mathbf{v}=$ $(u, w)$. The composition field $c=c_{1}-c_{2}$ represents the difference of concentration of the two components of the mixture, $p_{\text {eff }}=p-(c+1) \Delta c-(\Delta c)^{2} / 2$ is an effective pressure comprising all diagonal terms of the stress tensor, and $p$ is the 'normal' pressure. The local bulk free energy is assumed to correspond to the simple quartic potential $f(c)=\left(c^{2}-1\right)^{2} / 4$. To make the equations (11) and (2) dimensionless, in Ref. 37 the scales $l=C \sqrt{\sigma_{c} / E}, U=$ $M E / l C^{2}, \tau=l / U=l^{2} C^{2} /(M E)$ and $P=E$ are used for length, velocity, time and pressure respectively. The length $l$ corresponds to the thickness of the diffuse interface between the two phases of the mixture and is determined from the coefficient $\sigma_{c}$ of the gradient term of the convective Cahn-Hilliard equation (cf. Ref. 37), the energy scale $E$ and the concentration $C$ at the binodal. The parameter $M$ is the diffusion coefficient in the Cahn-Hilliard equation. With these scales, two dimensionless numbers appear in the bulk equations (11) and (2), the pressure number Ps $=\rho \mathrm{M}^{2} E^{2} / \mathrm{C}^{6} \sigma_{c}$ and the Reynolds number Re $=\mathrm{ME} \rho / \eta C^{2}$. Note that, as the diffusion constant is $D=M E$ and $U \sim D / l$, the used Reynolds number can be seen as an inverse Schmidt number $\mathrm{Sc}=\eta / \rho D$. The ratio $\mathrm{Re} / \mathrm{Ps}=\sigma_{c} C^{4} / \eta M E$ will turn out to be the most important bulk parameter for the extremely slow creeping flow we are interested in (see below Section $\llbracket$ IIIB). It corresponds to the ratio of the typical velocity $U=\sigma / \eta$ of the viscose flow driven by the internal 'diffuse interface tension' $\sigma=\sigma_{c} / l$ and the typical velocity of diffusive processes $U \sim M E / l=D / l$. This implies that Re/Ps can as well be seen as a Peclet number $\mathrm{Pe}=U^{\prime} l / D$ or as an inverse Capillary number $\mathrm{Ca}^{-1}=\sigma / U \eta$. Such alternative scalings are used, for instance, in Refs. 30 and 31 .

Equations (11) and (2) are supplemented with boundary conditions for the concentration and velocity fields. $\underline{37}$ We first present the conditions for the concentration field. At the solid substrate $(z=0)$ one prevents a diffusive mass flux through the substrate and allows for an energetic bias, i.e.,

$$
\begin{array}{r}
\partial_{z}\left[\left(\partial_{x x}+\partial_{z z}\right) c-\partial_{c} f(c)\right]=0 \\
-\partial_{z} c+\mathrm{S} \partial_{c} f^{-}(c)=0
\end{array}
$$

respectively. The energy bias is $f^{-}(c)=\gamma_{s}+a^{-} c+b^{-} c^{2} / 2$, where $\mathrm{S} \gamma_{s}$ is the dimensionless solid-liquid interface tension at $c=0$. Note that it does not influence later calculations as only $\partial_{c} f^{-}(c)$ enters. Parameters $a^{-}$and $b^{-}$model preferential adsorption of one of the species at the substrate and changes in the mixing/demixing behaviour of the species at the 
substrate, respectively. The dimensionless parameter $S=\gamma_{0} / l E$ is the dimensionless surface tension of the liquid-gas interface, and $\gamma_{0}$ is the reference surface tension at $c=0$. Similar conditions are applied at the (curved) free surface $(z=h(x, y, t))$, i.e.

$$
\begin{aligned}
{\left[\partial_{z}-\left(\partial_{x} h\right) \partial_{x}\right]\left[\left(\partial_{x x}+\partial_{z z}\right) c-\partial_{c} f(c)\right] } & =0 \\
{\left[\partial_{z}-\left(\partial_{x} h\right) \partial_{x}\right] c+\mathrm{S} \partial_{c} f^{+}(c) \sqrt{1+\left(\partial_{x} h\right)^{2}} } & =0
\end{aligned}
$$

with $f^{+}(c)=1+a^{+} c+b^{+} c^{2} / 2$. Parameters $a^{+}$and $b^{+}$quantify preferential adsorption of one of the species and changes in the the mixing/demixing behaviour at the free interface, respectively.

The boundary conditions for the velocity field are the no-slip and no-penetration condition at the substrate, i.e., $v=w=0$ at $z=0$. At the free surface the conditions result from the balance of tangential forces

$$
\begin{aligned}
& -\left[\partial_{x} c+\left(\partial_{x} h\right) \partial_{z} c\right]\left[\partial_{z} c-\left(\partial_{x} h\right) \partial_{x} c\right] \\
& +\frac{\operatorname{PS}}{\operatorname{Re}}\left[\left(u_{z}+w_{x}\right)\left(1-h_{x}^{2}\right)+2\left(w_{z}-u_{x}\right) h_{x}\right] \\
& =\mathrm{S} \sqrt{1+h_{x}^{2}}\left[\partial_{x}+\left(\partial_{x} h\right) \partial_{z}\right] f^{+}(c)
\end{aligned}
$$

and normal forces

$$
\begin{aligned}
& -\frac{1}{1+h_{x}^{2}}\left[\partial_{z} c-\left(\partial_{x} h\right) \partial_{x} c\right]^{2}-p_{\mathrm{eff}} \\
& +\frac{\operatorname{Ps}}{\operatorname{Re}} \frac{2}{1+h_{x}^{2}}\left[u_{x} h_{x}^{2}+w_{z}-h_{x}\left(u_{z}+w_{x}\right)\right] \\
& =\operatorname{Sf}^{+}(c) \partial_{x}\left[\frac{\partial_{x} h}{\left(1+h_{x}^{2}\right)^{1 / 2}}\right] .
\end{aligned}
$$

Notice that the tangential gradient of $f^{+}(c)$ enters the tangential force balance corresponding to a solutal Marangoni force. Furthermore, at the free surface one has a kinematic condition assuring that the free surface follows the velocity field

$$
\partial_{t} h=w-u \partial_{x} h
$$

After deriving the system of equations and boundary conditions, Ref. 37 embarks on an extensive study of quiescent, vertically homogeneous and vertically stratified base state solutions that are all homogeneous (or translationally invariant) with respect to the direction parallel to the substrate. The corresponding concentration profiles $c_{0}(z)$ are obtained by 
solving the steady bulk equation (steady version of Eq. (2))

$$
0=\partial_{z z}\left[\partial_{z z} c_{0}-\left.\partial_{c} f(c)\right|_{c_{0}}\right]
$$

with the boundary conditions

$$
0=\partial_{z}\left[\partial_{z z} c_{0}-\left.\partial_{c} f(c)\right|_{c_{0}}\right] \quad \text { at } \quad z=0, h_{0}
$$

and

$$
0= \pm \partial_{z} c_{0}+\left.\mathrm{S}_{c} f^{ \pm}(c)\right|_{c_{0}} \quad \text { at } \quad z=0, \quad h_{0}
$$

Here we are concerned with the stability of the base state concentration profiles $c_{0}(z)$ with respect to perturbations along the direction parallel to the substrate. The rational behind this approach is the experimental observation that thin films of a decomposing mixture first stratify vertically on a relatively short time scale and then develop on a slower time scale a horizontal structure. Here, we want to capture the characteristics of the latter process by the linear stability analysis.

\section{LINEARIZED EQUATIONS FOR VERTICALLY STRATIFIED AND HOMO- GENEOUS FILMS}

\section{General ansatz}

To analyze the stability of the quiescent base states with respect to infinitesimally small perturbations, we write the general solution of the problem in the form $\mathbf{v}=\mathbf{v}_{\mathbf{0}}+\varepsilon \tilde{\mathbf{v}}_{\mathbf{1}}$, $p_{\text {eff }}=p_{0}+\varepsilon \tilde{p}_{1}, c=c_{0}+\varepsilon \tilde{c}_{1}$, and $h=h_{0}+\varepsilon \tilde{h}_{1}$, with $\mathbf{v}_{\mathbf{0}}=0$ and $p_{0}=-\left(\partial_{z} c_{0}\right)^{2}$. The fields $\varepsilon \tilde{\mathbf{v}}_{\mathbf{1}}, \varepsilon \tilde{p}_{1}, \varepsilon \tilde{c}_{1}$, and $\varepsilon \tilde{h}_{1}$ denote the infinitesimal perturbations of velocity, pressure, concentration, and thickness fields, respectively. The small parameter $\varepsilon$ will be used to order terms in the series expansion. The perturbations are decomposed into a sum of normal modes $\left(\tilde{\mathbf{v}}_{\mathbf{1}}, \tilde{p}_{1}, \tilde{c}_{1}, \tilde{h}_{1}\right)=\left(\mathbf{v}_{1}(z), p_{1}(z), c_{1}(z), h_{1}\right) \exp (\beta t+i k x)$, where $\beta$ is the growth rate and $k$ the lateral wavenumber. Using this ansatz in Eqs. (11) to (10) we obtain the linearized convective Cahn-Hilliard equation

$$
\beta c_{1}+w_{1} \partial_{z} c_{0}=-\left(\partial_{z z}-k^{2}\right)\left[\left(\partial_{z z}-k^{2}\right) c_{1}-\left.c_{1} \partial_{c c} f\right|_{c_{0}}\right]
$$


and the linearized momentum equation

$$
\begin{aligned}
\beta \operatorname{Ps} \mathbf{v}_{1}= & -\nabla \cdot\left\{\left[\left(\nabla c_{0}\right)\left(\nabla c_{1}\right)+\left(\nabla c_{1}\right)\left(\nabla c_{0}\right)\right]+p_{1} \underline{\mathbf{I}}\right\} \\
& +\frac{\operatorname{Ps}}{\operatorname{Re}}\left(-k^{2}+\partial_{z z}\right) \mathbf{v}_{1}
\end{aligned}
$$

where the tensor $\left[\left(\nabla c_{0}\right)\left(\nabla c_{1}\right)+\left(\nabla c_{1}\right)\left(\nabla c_{0}\right)\right]=\left[\left(0, i k c_{1} \partial_{z} c_{0}\right),\left(i k c_{1} \partial_{z} c_{0}, 2\left(\partial_{z} c_{1}\right)\left(\partial_{z} c_{0}\right)\right)\right]$, i.e. splitting the velocity field in its components we obtain

$$
\begin{aligned}
\beta \operatorname{Ps} u_{1}= & -i k \partial_{z}\left(c_{1} \partial_{z} c_{0}\right)-i k p_{1} \\
& +\frac{\operatorname{Ps}}{\operatorname{Re}}\left(-k^{2}+\partial_{z z}\right) u_{1} \\
\beta \operatorname{Ps} w_{1}= & -\left[-k^{2} c_{1} \partial_{z} c_{0}+2 \partial_{z}\left(\left(\partial_{z} c_{0}\right)\left(\partial_{z} c_{1}\right)\right)\right]-\partial_{z} p_{1} \\
& +\frac{\operatorname{Ps}}{\operatorname{Re}}\left(-k^{2}+\partial_{z z}\right) w_{1} .
\end{aligned}
$$

The incompressibility condition leads to

$$
i k u_{1}+\partial_{z} w_{1}=0
$$

The linearized boundary conditions assuring zero mass flux through the substrate at $z=0$ and through the free surface at $z=h_{0}$ are both of the form

$$
0=\partial_{z}\left[\left(-k^{2}+\partial_{z z}\right) c_{1}-\left.c_{1} \partial_{c c} f\right|_{c_{0}}\right]
$$

The linearized energy bias conditions for the concentration are

$$
\begin{aligned}
&-\partial_{z} c_{1}+\left.\mathrm{S} c_{1} \partial_{c c} f^{-}\right|_{c_{0}}=0 \text { at } z=0 \\
& \text { and } \partial_{z} c_{1}+\left.\mathrm{S} c_{1} \partial_{c c} f^{+}\right|_{c_{0}}=0 \text { at } z=h_{0} .
\end{aligned}
$$

The non-slip condition for the velocity at the substrate becomes

$$
u_{1}=w_{1}=0
$$

and the linearized tangential and normal force balance at the free interface are

$$
\begin{aligned}
& \frac{\mathrm{PS}}{\operatorname{Re}}\left(\partial_{z} u_{1}+i k w_{1}\right)=0, \\
& 2\left(\partial_{z} c_{0}\right)\left(\partial_{z} c_{1}\right)+p_{1}-2 \frac{\mathrm{PS}}{\operatorname{Re}} \partial_{z} w_{1}=\left.\mathrm{S} f^{+}\right|_{c_{0}} k^{2} h_{1},
\end{aligned}
$$

respectively. Finally, the kinematic condition reads

$$
w_{1}=\beta h_{1}
$$


Note that the r.h.s. of Eq. (23) is exactly zero following from the boundary condition Eq. (13) of the base state. Physically this means that the Korteweg and the Marangoni stress at the free surface exactly compensate, i.e. all the tangential driving force is already contained in the linearized bulk equation. The situation would be different if we were to allow for a dynamic surface tension different from the used static one.

\section{Eigenvalue problem for stratified film}

To carry out the stability analysis of the linearized model-H we eliminate the pressure in the momentum equation and boundary conditions, and write the linearized model- $\mathrm{H}$ as an eigenvalue problem of the form

$$
\begin{aligned}
\partial_{z z z z} c_{1} & =-\left(\beta c_{1}+w_{1} \partial_{z} c_{0}\right) \\
& -\left[\left(k^{4}-2 k^{2} \partial_{z z}\right) c_{1}-\left(\partial_{z z}-k^{2}\right)\left(\left.c_{1} \partial_{c c} f\right|_{c_{0}}\right)\right]
\end{aligned}
$$

and

$$
\begin{aligned}
\partial_{z z z z} w_{1} & =\beta \operatorname{Re}\left(\partial_{z z}-k^{2}\right) w_{1}+\frac{\operatorname{Re}}{\operatorname{Ps}} k^{4} c_{1} \partial_{z} c_{0} \\
& -\frac{\operatorname{Re}}{\operatorname{Ps}} 2 k^{2} \partial_{z}\left[\left(\partial_{z} c_{0}\right)\left(\partial_{z} c_{1}\right)\right] \\
& +\frac{\operatorname{Re}}{\operatorname{Ps}} k^{2} \partial_{z z}\left(c_{1} \partial_{z} c_{0}\right)+2 k^{2} \partial_{z z} w_{1}-k^{4} w_{1}
\end{aligned}
$$

with the boundary conditions

$$
\begin{gathered}
w_{1}=\partial_{z} w_{1}=0 \quad \text { at } \quad z=0 ; \\
\frac{\operatorname{Ps}}{\operatorname{Re}}\left(\partial_{z z} w_{1}+k^{2} w_{1}\right)=0 \quad \text { at } \quad z=h_{0} ;
\end{gathered}
$$

and

$$
\begin{aligned}
& k^{2}\left(\partial_{z} c_{0}\right)\left(\partial_{z} c_{1}\right)-k^{2} c_{1} \partial_{z}^{2} c_{0}+\frac{\operatorname{Ps}}{\operatorname{Re}}\left(\partial_{z z}-3 k^{2}\right) \partial_{z} w_{1} \\
& -\beta \operatorname{Ps} \partial_{z} w_{1}=\left.\mathrm{Sf}^{+}\right|_{c_{0}} k^{4} h_{1} \quad \text { at } \quad z=h_{0} ;
\end{aligned}
$$

where $h_{1}=w_{1} / \beta$. Furthermore,

$$
\begin{gathered}
\partial_{z}\left[\left(-k^{2}+\partial_{z z}\right) c_{1}-\left.c_{1} \partial_{c c} f\right|_{c_{0}}\right]=0 \quad \text { at } \quad z=0, h_{0} \\
-\partial_{z} c_{1}+\left.\mathrm{S} c_{1} \partial_{c c} f^{-}\right|_{c_{0}}=0 \quad \text { at } \quad z=0 \\
\partial_{z} c_{1}+\left.\mathrm{S} c_{1} \partial_{c c} f^{+}\right|_{c_{0}}=0 \quad \text { at } \quad z=h_{0} .
\end{gathered}
$$




\section{Eigenvalue problem for homogeneous films}

For homogeneous films the concentration does not depend on $z$, i.e., the base state is uniform along both, the $x$ - and $z$-direction. In consequence, the eigenvalue problem reduces to the bulk equations

$$
\partial_{z z z z} c_{1}=-\beta c_{1}-\left(k^{4}-2 k^{2} \partial_{z z}\right) c_{1}-\left(\partial_{z z}-k^{2}\right)\left(\left.c_{1} \partial_{c c} f\right|_{c_{0}}\right)
$$

and

$$
\partial_{z z z z} w_{1}=\beta \operatorname{Re}\left(\partial_{z z}-k^{2}\right) w_{1}+2 k^{2} \partial_{z z} w_{1}-k^{4} w_{1}
$$

with the boundary conditions

$$
\begin{gathered}
w_{1}=\partial_{z} w_{1}=0 \text { at } z=0 ; \\
\frac{\operatorname{Ps}}{\operatorname{Re}}\left(\partial_{z z} w_{1}+k^{2} w_{1}\right)=0 \quad \text { at } \quad z=h_{0} ; \\
\frac{\operatorname{Ps}}{\operatorname{Re}}\left(\partial_{z z}-k^{2}\right) \partial_{z} w_{1}-\beta \operatorname{Ps} \partial_{z} w_{1}-\frac{\operatorname{Ps}}{\operatorname{Re}} 2 k^{2} \partial_{z} w_{1} \\
=\left.\mathrm{S} f^{+}\right|_{c_{0}} k^{4} w_{1} / \beta \text { at } z=h_{0} ; \\
\partial_{z}\left[\left(-k^{2}+\partial_{z z}\right) c_{1}-\left.c_{1} \partial_{c c} f\right|_{c_{0}}\right]=0 \quad \text { at } z=0, h_{0} ; \\
-\partial_{z} c_{1}+\left.\mathrm{S} c_{1} \partial_{c c} f^{-}\right|_{c_{0}}=0 \quad \text { at } z=0 ; \\
\text { and } \partial_{z} c_{1}+\left.\mathrm{S} c_{1} \partial_{c c} f^{+}\right|_{c_{0}}=0 \text { at } z=h_{0} .
\end{gathered}
$$

Inspection of the equations shows that the perturbations in the concentration and in the velocities do decouple entirely, i.e., the linear stability problem for the concentration field in a homogeneous film reduces to the one resulting from the Cahn-Hilliard equation in a slab ${ }^{38}$. The decoupled velocity perturbations are all damped out.

\section{A. Numerical technique}

In the present work the calculations of the base states and their linear stability are carried out using numerical continuation techniques bundled in the package AUTO $\underline{39} \underline{-41}$.

Continuation techniques allow to obtain solutions of a problem for a given set of control parameters by 'extrapolation' from known solutions that are nearby in the parameter space. 
In particular, the set of equations for the steady state (111) and the for real linear perturbations (26)-(27) can be written as a 10-dimensional dynamical system $\mathbf{y}^{\prime}(z)=\mathbf{f}(\mathbf{y}(z), \lambda)$, where $\mathbf{y}=\left(c_{0}, c_{0 z}, c_{1}, c_{1 z}, c_{1 z z}, c_{1 z z z}, w_{1}, w_{1 z}, w_{1 z z}, w_{1 z z z}\right)$ and $\lambda$ denotes a set of control parameters (in our case Re, $\mathrm{Ps} / \mathrm{Re}, \mathrm{S}, \beta, k, \bar{c}$ ). This system of ordinary differential equations together with the boundary conditions at $z=0$ and $z=h$ (28)-(33) and one integral condition (mass conservation) is discretized in space and the resulting algebraic system is solved iteratively. The package AUTO ${ }^{39}$ uses the method of orthogonal collocation to discretize solutions, where the solution is approximated by piecewise polynomials with two collocation points per mesh interval. The mesh is adaptive to equidistribute the discretization error. Starting from known solutions, AUTO tries to find nearby solutions to the discretized system by using a combination of Newton and Chord iterative methods. Once the solution has converged, AUTO proceeds along the solution branch by a small step in the parameter space defined by the free continuation parameters and restarts the iteration. Boundary conditions and integral conditions require additional free parameters which are determined simultaneously and are part of the solution of the differential equation. The package AUTO is limited to the continuation of ordinary differential equations, and has been successfully applied to other thin film problems like, e.g., the determination of dispersion relations for transverse instabilities of advancing liquid fronts and ridges $\frac{42}{2}$; the determination of steady and stationary thickness profiles and their linear stability for sliding $\underline{43}-\underline{45}$, running $\underline{46}$ or depinning $\underline{47}$ droplets; the analysis of steady film profiles for epitaxial growth플 in falling film problems $\underline{49}-\underline{51}$.

The main difficulty is usually to provide a starting solution for the continuations. Here, we follow different sequences of steps starting from trivial analytically known solutions. For $n=0$ branches (for definition see below) we (i) increase the thickness starting from a homogeneous solution of small thickness, (ii) increase the energetic bias at the surfaces if needed, (iii) look for branching points when increasing the linear growth rate for some fixed wave number, (iv) calculate the dispersion relation and its maximum, (v) and follow the maximum in a multi-parameter continuation. For $n \neq 0$ branches we (i) start with some fixed thickness, increase the energetic bias at the surface if needed and identify branching points where non-trivial solutions emerge (ii) increase the thickness following the various emerging branches, (iii)-(v) as above. In this way the linear stability of the various stratified films can be determined in a rather effective manner. 


\section{B. Parameter range}

Due to the large number of dimensionless parameters needed to describe a film of a binary mixture with a free surface, we proceed to discuss the stability results in a restricted range of parameters that is of particular experimental interest. For instance, the polymer blend Polystyrene/Polyvinylmethylether (PS/PVME) has been widely used in thin film experiments, without $\underline{52,53}$ and with $\underline{53}$ the presence of an external electric field. We will use this mixture as a reference for our calculations. The density of polystyrene at $T=170^{\circ} \mathrm{C}$ is $\rho \approx 0.987 \cdot 10^{3} \mathrm{~kg} / \mathrm{m}^{3}$, its viscosity is $\eta=4062 \mathrm{~kg} / \mathrm{ms}$, and its surface tension is $\gamma_{0}=0.03$ $\mathrm{N} / \mathrm{m}$. The surface tension of Polyvinylmethylether is $\gamma_{0}=0.021 \mathrm{~N} / \mathrm{m}$. A linear estimate of the variation of the surface tension of the mixture with the concentration gives $d \gamma / d c=0.018$ $\mathrm{N} / \mathrm{m} \cdot \underline{\underline{53}}$

To calculate the coefficients of the free energy $a$ and $b, \underline{54}$ and the coefficient of the gradient term in the Cahn-Hilliard equation $\sigma_{c}$ (cf. Ref. 37 for a more detailed discussion of the coefficients), we use the Flory-Huggins mode ${ }^{55,56}$. In this model one supposes that the monomers of the chains of the two polymers composing the binary mixture occupy different sites on a square lattice. For polystyrene molecules with typically 1000 monomers, and a lattice spacing of $\sim 10^{-10} \mathrm{~m}$ the random phase approximation $\underline{\underline{56}} \underline{\underline{58}}$ gives $\sigma_{c} \sim 10^{-16}$ at $T=170^{\circ} C \underline{\underline{59}}$ This finally allows us to estimate $a=-\mathcal{O}(1)$ and $b=\mathcal{O}(1)$. The scale of the concentration field $C$ must be $\leq \mathcal{O}(1)$ and we consider $C \sim 0.5$, consistent with $C=\sqrt{a / b}$.

The diffusion coefficients for polymers measured in experiments range from $10^{-17}$ to $10^{-19} \mathrm{~cm}^{2} / \mathrm{s} \cdot \underline{\underline{60}} \underline{\underline{63}}$ We choose here the value obtained by Reiter and Steiner $\underline{\underline{64}}$ of $D \sim$ $10^{-17} \mathrm{~cm}^{2} / \mathrm{s}$ because their case is close to ours. The mobility coefficient of the Cahn-Hilliard equation $M$ is calculated according to the relation $M=D / E$ with the characteristic energy per unit volume $E=4 a^{2} / b$ and the characteristic diffusivity $D$. We find $M \sim 10^{-20} \mathrm{~m}^{3} \mathrm{~s} / \mathrm{kg}$. The obtained parameters correspond to a characteristic time scale of a few hundredths of a second and a characteristic length scale of some nanometers. From the estimates of the physical parameters we obtain the dimensionless parameters $\operatorname{Re} \simeq 10^{-17}$ and $\mathrm{Ps} \simeq 10^{-15}$. Therefore, we set $\mathrm{Re}=\mathrm{Ps}=0$ but keep the ratio $\mathrm{Re} / \mathrm{Ps}$ as an important parameter of order one. Normally, we will keep Re/Ps= 1 fixed but we investigate its influence below in Section $\mathrm{VC}$. These values correspond to the fact that polymers flow in an extremely slow creeping flow as expected. However, as explained before that flow has to be taken into ac- 
count in order to explain and describe the evolution of the film profile. In consequence, the only free parameter that enters the bulk equations (14) and (15) is the ratio Re/Ps. Next we discuss the dimensionless numbers that enter the boundary conditions. For the surface tension number $\mathrm{S}$, we obtain $\mathrm{S} \simeq 10^{3}$. However, it depends linearly on the interfacial tension and therefore on the type of polymers of the binary mixture. Therefore we will allow it to vary. Finally, $a^{+}, a^{-}, b^{+}$, and $b^{-}$depend on the nature of the surfaces and therefore we will treat them as free surface parameters.

From the original large number of parameters we, finally retain as free parameters for realistic binary mixtures the time scale ratio Re/Ps important for the bulk flow, and the numbers related to surface properties $a^{ \pm}, b^{ \pm}$and surface tension number S. The remaining dimensionless numbers discussed above or in Ref. 37 are either very small, considered as zero for all purposes, or equal to one because of the scaling used.

\section{LINEAR STABILITY OF HOMOGENEOUS FILMS}

Homogeneous base states are characterized by a concentration $c_{0}$ constant across the sample and a quiescent fluid $\mathbf{v}_{\mathbf{0}}=0$. Arbitrary concentrations $c_{0}$ are possible for neutral surfaces $a^{ \pm}=b^{ \pm}=0$. An energetic bias at the surfaces leads to the restriction $c_{0}=$ $-a^{+} / b^{+}=-a^{-} / b^{-}$(see Ref. 37 ) whenever $b^{ \pm} \neq 0$.

Inspecting Eqs. (34) to (41) we note that the evolution of the perturbation of the concentration field $c_{1}$ is independent of the perturbation of the velocity field $w_{1}$, i.e., the perturbation fields $c_{1}$ and $w_{1}$ are neither coupled in the bulk equations nor in the boundary conditions. This decoupling of $w_{1}$ and $c_{1}$ results in $w_{1}=0$ and implies that hydrodynamics has no influence on the evolution of the homogeneous film in the linear stage. A further consequence is the lack of surface deflection defined as $h_{1}=w_{1} / \beta$.

The decoupling of velocity and concentration fields implies that all unstable modes are purely diffusive and correspond to the ones that can be obtained in a model based solely on the Cahn-Hilliard equation. We stress again that no surface deflection can develop. In the following we study the diffusive modes to put the present results in the context of results obtained in the literature. 


\section{A. Neutral surfaces $a^{ \pm}=b^{ \pm}=0$}

Neutral surfaces correspond to the simplest possible configuration for a binary film. The bounding substrate and the free surface do not prefer attachment by any component. They do neither influence mixing or demixing phenomena. They only confine the film passively. Thereby, however, they restrict the possible instability modes as compared to the bulk. From Eq. (34) we obtain the dispersion relation for neutral surfaces

$$
\beta=-q^{2}\left(q^{2}+\left.\partial_{c c} f(c)\right|_{c_{0}}\right)
$$

with $q^{2}=k^{2}+k_{z}^{2}$ and the vertical eigenfunctions are of the form $\sim \exp \left(i k_{z} z\right)$. For offcritical mixtures (i.e., $\left.c_{0} \neq 0\right)\left.\partial_{c c} f(c)\right|_{c_{0}}=3 c_{0}^{2}-1$, i.e., spinodal decomposition only occurs for $\left|c_{0}\right|<1 / \sqrt{3}$. Here, we entirely focus on critical mixtures $\left(c_{0}=0\right)$ with $\left.\partial_{c c} f(c)\right|_{c_{0}}=$ -1 . As the system is infinitely extended in $x$-direction the horizontal wavenumber $k$ is continuous. However, the vertical wavenumber can only take discrete values $k_{z}=2 \pi m / h$ where $m=0,1 / 2,1, \ldots$ is a mode number. The values $0,1 / 2$ and 1 correspond to a vertically homogeneous mode, a vertical two-layer mode and a vertical three-layer sandwich mode, respectively.

The $m=0$ mode is characterized by a purely horizontal variation of the concentration field $c_{1}$ (cf. Fig. 1(a)) without any vertical structure $\left(k_{z}=0\right)$. For neutral surfaces it is present for all film thicknesses. The corresponding dispersion relation $\beta_{0}=-k^{2}\left(k^{2}-1\right)$ is shown in Fig. 1(a). The maximum growth rate is $\beta_{\max }^{m=0}=1 / 4$ at $k_{\max }=1 / \sqrt{2}$. Due to mass conservation $\beta=0$ for $k=0$. The mode corresponds to the long-wave bulk mode of the Cahn-Hilliard equation. 65,66

The first mode with a vertical structure is the $m=1 / 2$ mode. It is unstable for $h>\pi$. For $h \leq \sqrt{2} \pi$ and $h>\sqrt{2} \pi$ the mode has the dispersion relations presented in Figs. 1(b) and 1(c), respectively. For $h \leq \sqrt{2} \pi$ the mode shows its maximum growth rate $\beta_{\max }^{m=1 / 2}$ at zero horizontal wavenumber $k=0$, i.e., it is purely vertical. Note that for neutral surfaces $\beta_{\max }^{m=1 / 2}$ is always smaller than $\beta_{\max }^{m=0}$ (equal at $h=\sqrt{2} \pi$, see Fig. 2(a)). For $h>\sqrt{2} \pi$ the $m=1 / 2$ mode has a dispersion relation shown in Fig. 1(c) that we call 'mixed mode' because one has a vertical as well as a horizontal structuring (checkerboard-like) of the unstable film because $m>0$ and $k_{\max } \neq 0$. Note as well that $\beta>0$ at $k=0$ and $\beta_{\max }=1 / 4$ as for the $m=0$ mode. As $h$ increases the fastest growing horizontal wavenumber of the mixed mode becomes larger (Fig. 2(b)) and approaches the one of the horizontal mode whereas its 


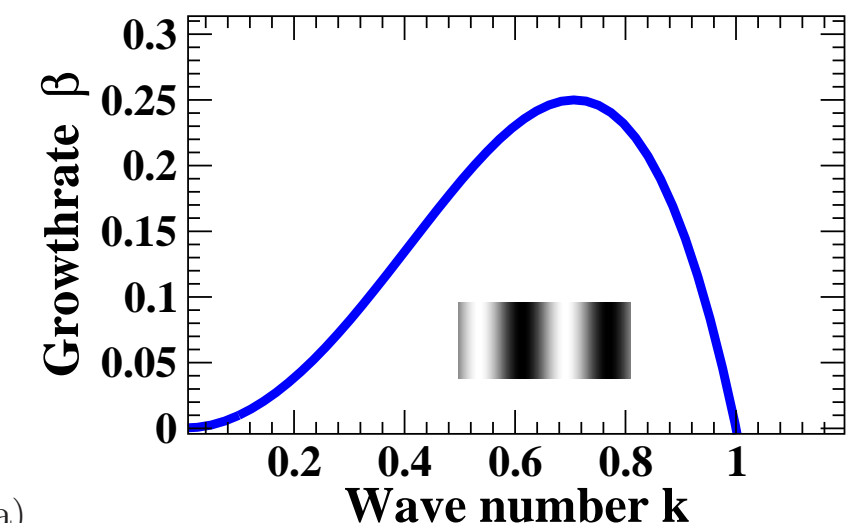

(a)

(b)

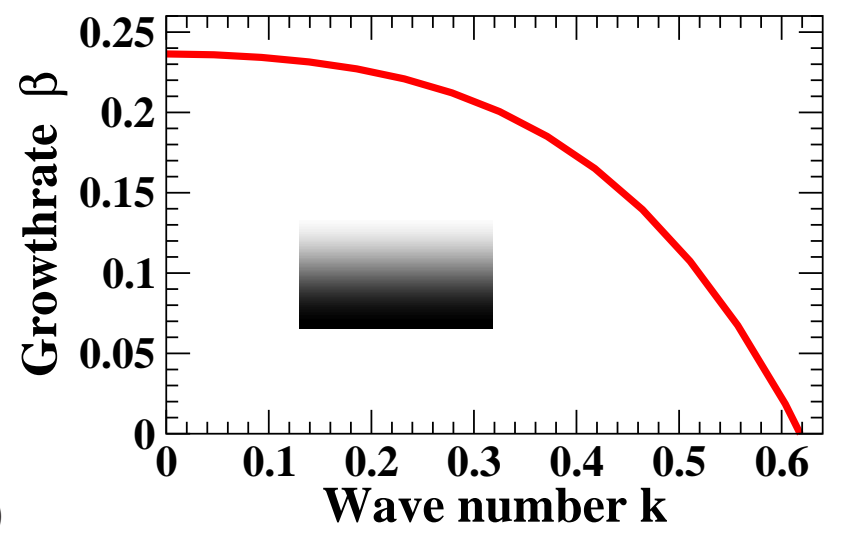

(c)

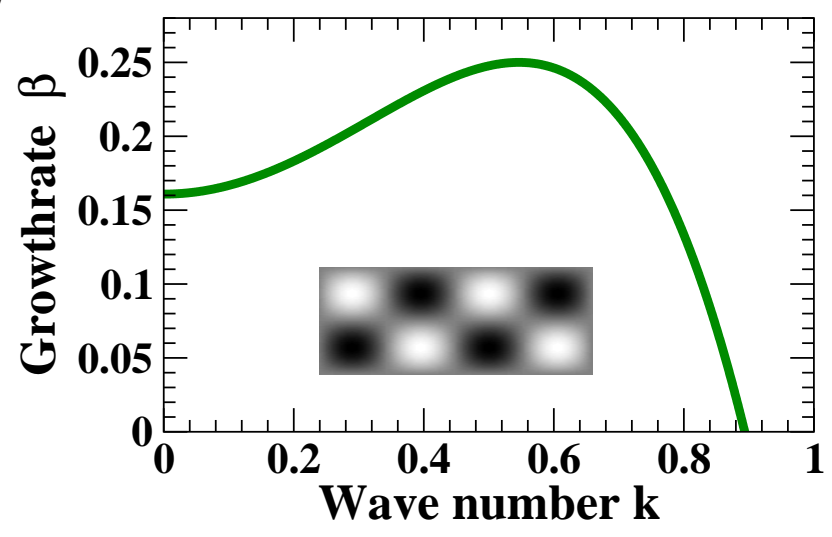

Figure 1: (color online) Selected dispersion relations for a homogeneous film of a critical mixture $c_{0}=0$ with energetically neutral surfaces $a^{ \pm}=b^{ \pm}=0$ and thicknesses (a) $h=3$ (horizontal mode), (b) $h=4$ (vertical mode), and (c) $h=7$ (mixed mode). The insets show sketches of the evolving concentration patterns for the corresponding modes. The dispersion relations discussed below for homogeneous films with energetically biased surfaces and as well for stratified films have similar shapes and will not be shown. 
growth rate equals $\beta_{\max }^{m=0}=1 / 4$. The minimum of the dispersion relation at $k=0$ becomes deeper as $h$ increases (thin dashed line in Fig. 2(a)).

Higher order modes $m \geq 1$ destabilize successively at $h=2 m \pi$. The resulting instability correspond to purely vertical modes with $\beta_{\max } \leq 1 / 4$ for $h \leq 2 \sqrt{2} m \pi$, and to mixed modes with $\beta_{\max }=1 / 4$ for $h>2 \sqrt{2} m \pi$.

Note, finally, that the dispersion relations that will be discussed below for homogeneous films with energetically biased surfaces and for stratified films have similar shapes as the ones in Figs. 1(a) to 1(c). However, they do not fall into the classification of modes used in this section.

\section{B. Energetically biased surfaces $a^{ \pm}=0, b^{ \pm} \neq 0$}

The relevance of energetically biased surfaces in the mixing/demixing dynamics has been recognized in the design of materials based on polymer blends $\frac{56}{}$. A biased surface can cause the formation of spinodal composition waves in a direction normal to the surfaces 23,24 giving rise to the so-called surface directed spinodal decomposition 24 .

According to the expression of the surface free energies $f^{+}(c)=1+a^{+} c+b^{+} c^{2} / 2$ and $f^{-}(c)=\gamma_{s}+a^{-} c+b^{-} c^{2} / 2$ and the boundary conditions (38), (40), and (41), the influence of the surfaces enters the stability problem through the coefficients $a^{ \pm}$that account for a preferential adsorption of one of the components at the respective surface, and the coefficients $b^{ \pm}$that describe mixing/demixing properties at the surfaces that differ from the bulk.

An energetic bias at the surfaces leads to the restriction $c_{0}=-a^{+} / b^{+}=-a^{-} / b^{-}$for the mean concentration. 37 It is very unlikely that the general case can be easily realized in an experiment. Therefore we restrict our analysis for the homogeneous film to the case $b^{ \pm} \neq 0$ with $a^{ \pm}=0$. Experimental realization seems as well questionable, however, the case is more generic as a critical mixture yields a valid base state for any $b^{+}$and $b^{-}$. The case was studied in depth in Ref. 67 in the context of purely diffusive decomposition of a binary mixture in a gap between two solid plates. This allows us to use the case as a benchmark for our numerical procedure by comparing our results with the literature.

We introduce a number of archetypal cases for $b^{ \pm}$: the symmetric case with $b^{+}=b^{-}$ where both surfaces of the film have identical bias; the antisymmetric case $b^{+}=-b^{-}$, where the two surfaces have opposite bias; and the asymmetric case where either $b^{+}$or $b^{-}$is zero. 


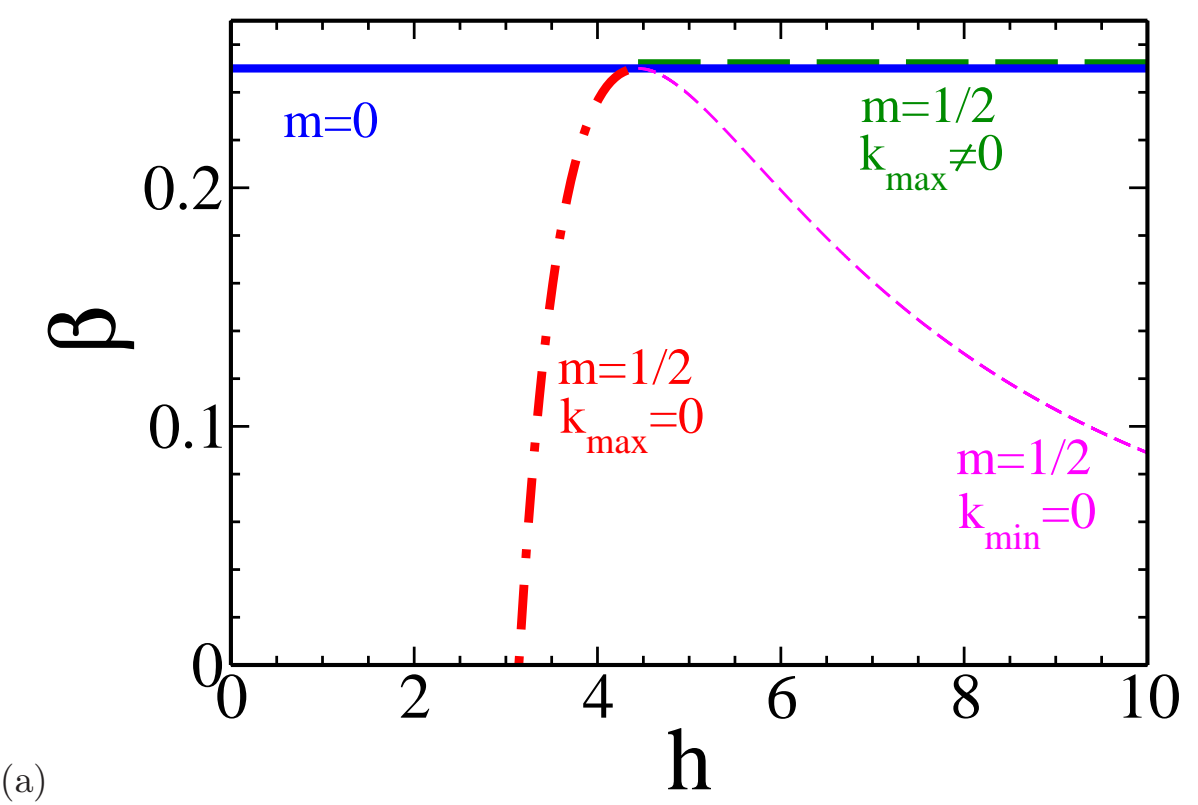

(a)

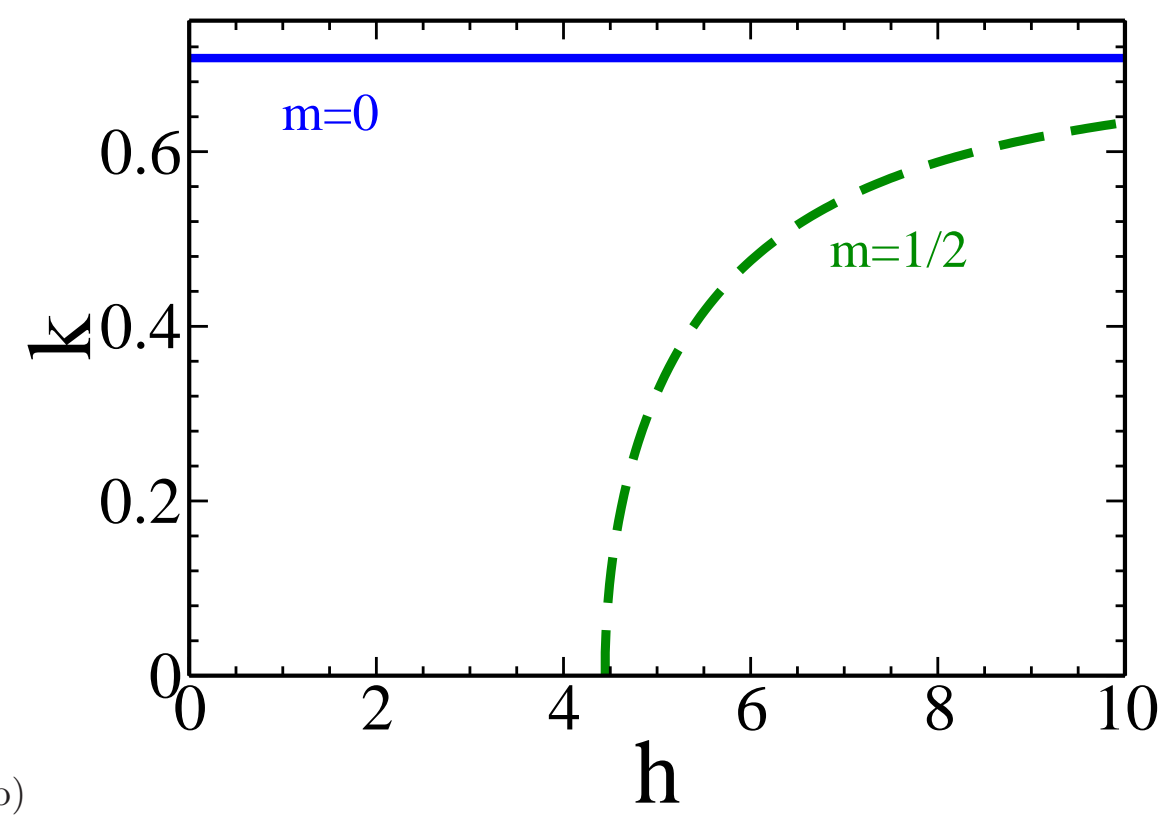

Figure 2: (color online) Maximum growth rate (a) and corresponding wavenumber (b) as a function of the thickness for a homogeneous film of critical composition $c_{0}=0$ and neutral surfaces $a^{ \pm}=0$ and $b^{ \pm}=0$. Solid line: mode $m=0$ (Fig. 1(a)). Dot-dashed line: mode $m=1 / 2$ with $k_{\max }=0$ (Fig. 1(b)). Dashed line: mode $m=1 / 2$ with $k_{\max } \neq 0$ (Fig. 1(c)). Thin dashed-line: growth rate $\beta$ of the mixed mode $m=1 / 2$ at the minimum of dispersion relation at $k_{\min }=0$. 
Fig. 3 presents typical results for the two most dangerous transverse instability modes for the symmetric $\left(b^{+}=b^{-}=1\right.$, top row $)$, asymmetric $\left(b^{+}=1, b^{-}=0\right.$, middle row $)$, and antisymmetric $\left(b^{+}=-b^{-}=1\right.$, bottom row) case. A selection of the corresponding eigenmodes $c_{1}(z)$ is given in Fig. 4(a) to (c), respectively.

In all cases one of the two most dangerous modes has a dispersion relation similar to Fig. 1(a), i.e., $\beta_{\max } \rightarrow 0$ for $k_{\max } \rightarrow 0$. It is nevertheless not a purely 'horizontal mode' as the eigenfunction $c_{1}$ shows a clear vertical structure (see Fig. 4). It dominates for symmetric bias (Fig. 3(a)). The other mode has normally a dispersion relation similar to Fig. 1(c), i.e., $\beta_{\max } \neq 0$ at $k_{\max }=0$. However, for $4.8<h<6$ in the symmetric case $\beta_{\max }$ occurs at $k_{\max }=0$ (analogous to Fig. 1(b)). This mode dominates for asymmetric and antisymmetric bias (Fig. 3(b) and Fig. 33(c), respectively).

Inspecting Fig. 3 one notes that for symmetric and asymmetric bias the dependency on film thickness is similar to the one for the $m=1 / 2$ mode in the case of neutral surfaces (Fig. 2): All modes stabilize at a thickness $h_{c}$, wavenumber and growth rate increase with increasing $h>h_{c}$ and for large $h$ both $\beta_{\max }$ approach $1 / 4$. The convergence at large $h$ is expected as the relative influence of the walls decreases with increasing $h$. Below $h_{c}$ the film is linearly stable.

For antisymmetric surface bias (bottom row of Fig. 3) one of the modes shows a similar behavior as the above modes. The other one, however, becomes progressively more unstable as $b^{-}$decreases (as compared to the asymmetric case). The growth rate converges for large thicknesses to a value much larger than the bulk value of $1 / 4$. The corresponding eigenmode given in Fig. 4(c) indicates that the film remains nearly homogeneous at the top where $b^{+}=1$ suppresses the demixing. At the bottom demixing is enforced by $b^{-}=-1$ and becomes much stronger than bulk demixing.

\section{RESULTS FOR STRATIFIED BASE STATES}

Non-trivial quiescent horizontally homogeneous base states of model-H are vertically nonuniform solutions, i.e., layered films. They are characterized by $z$-dependent concentration profiles $c_{0}=c_{0}(z)$ and $\mathbf{v}_{\mathbf{0}}=0$. In part $\mathrm{I}^{37}$, we discuss for different energetic biases at the surfaces various types of stratified film states in dependence of film thickness $h$. Several branches of stratified solutions may co-exist. They can be ordered by the mode type of 

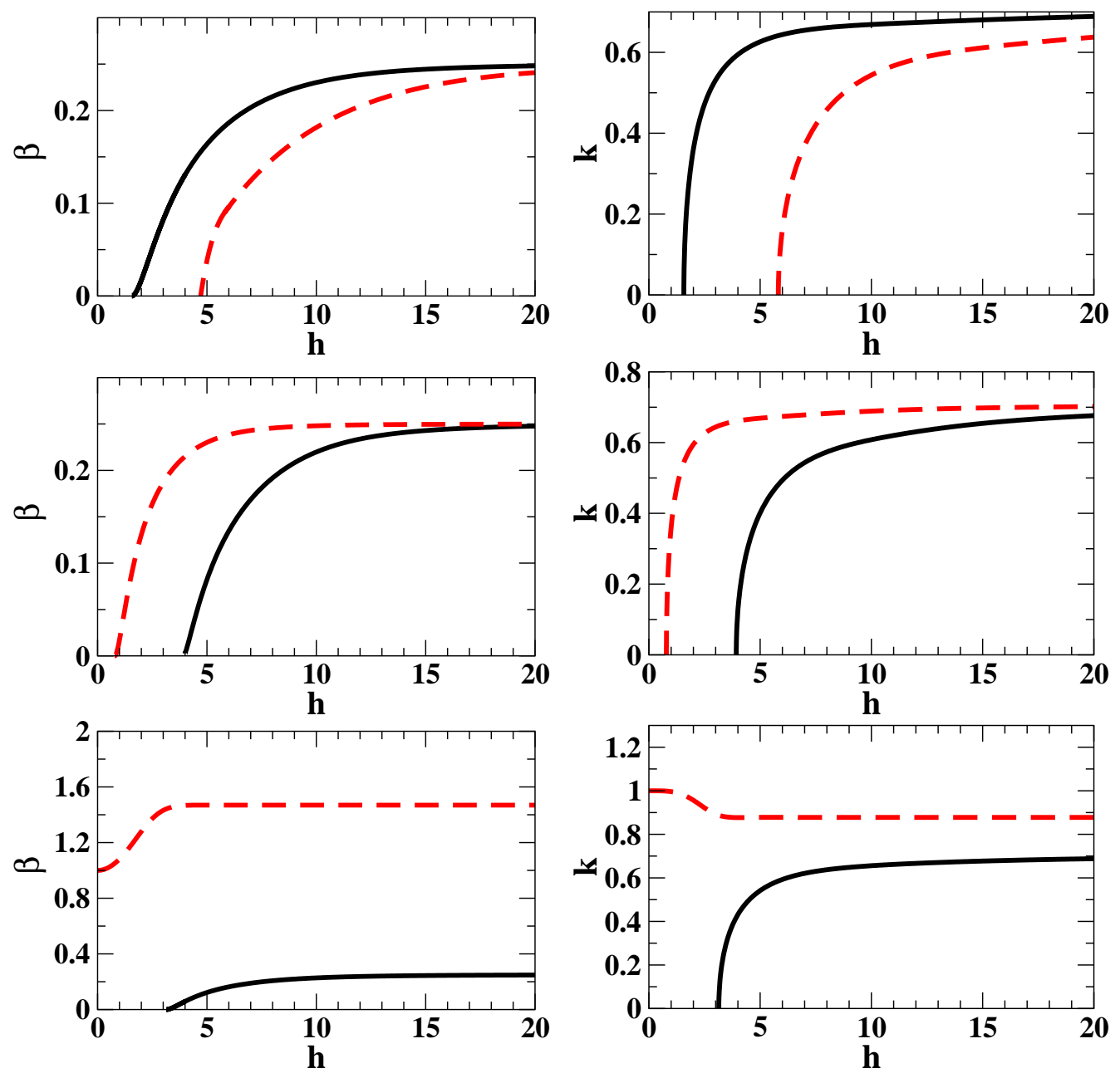

Figure 3: (color online) Maximum growth rate (left) and corresponding wavenumber (right) as a function of the thickness for an homogeneous film $c_{0}=0$ with biased surfaces. Top row: symmetric bias with $b^{ \pm}=1$ disfavoring demixing at both surfaces; middle row: asymmetric bias with $b^{+}=1$ and $b^{-}=0$ disfavoring demixing at the top surface; bottom row: antisymmetric bias with $b^{+}=1$ and $b^{-}=-1$ disfavoring (favoring) demixing at the top (bottom) surface. All solid lines correspond to maxima of dispersion relations similar to the one illustrated in Fig. 1(a), whereas dashed lines normally correspond to relations similar to the one in Fig. 1(c).

the related linear instability mode, i.e., the instability mode that destabilizes the trivial homogeneous solution at the bifurcation of the respective branch of stratified states. For details see Ref. 37 .

Although the pattern of branching is intriguing and interesting from the point of view of bifurcation theory, only a subset of branches has a large importance for experimental 


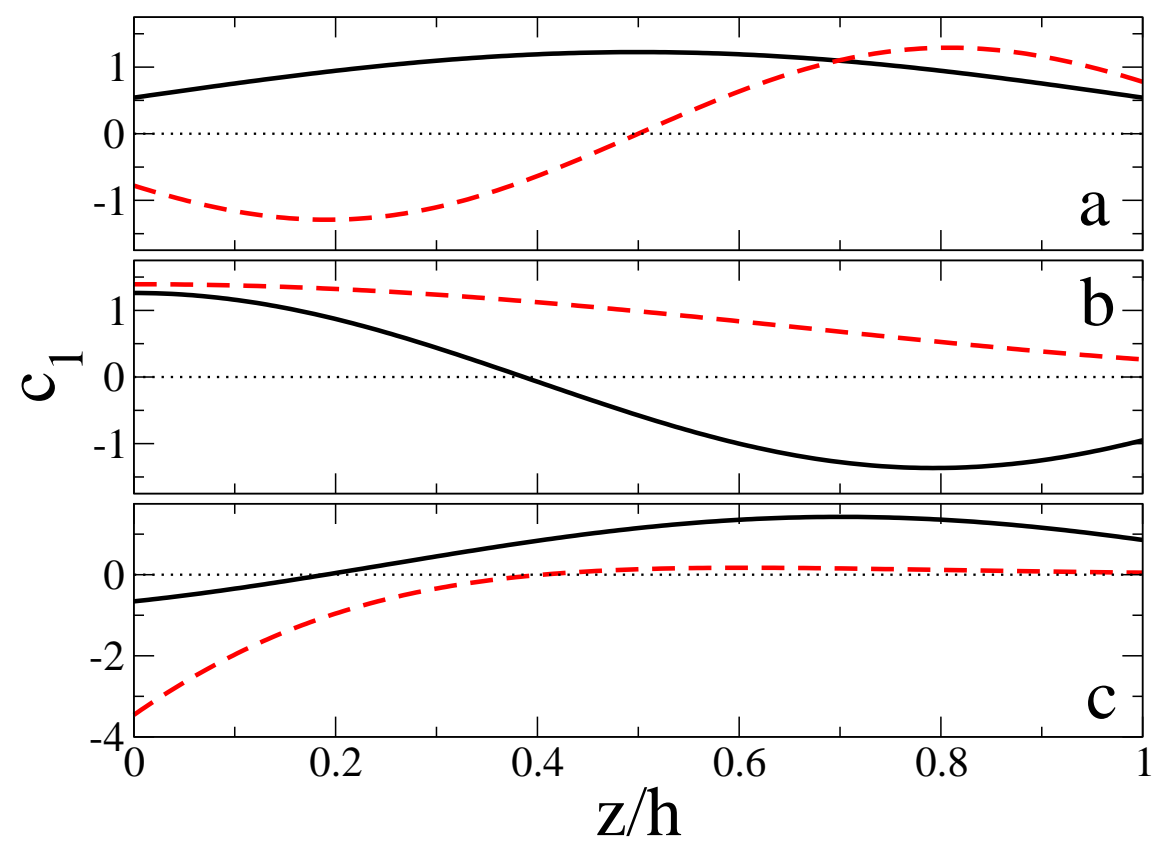

Figure 4: (color online) Selection of eigenmodes $c_{1}(z)$ for fixed thickness $h_{0}=5$ corresponding to the instability modes presented in Fig. 3. (a) symmetric bias with $b^{ \pm}=1$; (b) asymmetric bias with $b^{+}=1$ and $b^{-}=0 ;\left(\right.$ c) antisymmetric bias with $b^{+}=1$ and $b^{-}=-1$. Solid lines correspond to maxima of dispersion relations similar to the one illustrated in Fig. 1(a), whereas dashed lines correspond to relations similar to the one in Fig. 1(c).

systems. They can be identified calculating their energy (cf. Figs. 4, 8 and 10 of Ref. 37). The figures show that depending on parameter values the stratified films of either type $n=0$ or $n=1 / 2$ correspond to the energetic minimum. For weak surface bias, these solutions correspond roughly to a weak vertical stratification for $n=0$ and to strongly stratified two-layer configurations for $n=1 / 2$. For stronger bias they might as well correspond to a 3-layer sandwich configuration. In the following, we restrict our attention to the lateral stability of the solutions that are energetically most favorable.

\section{A. Stability without hydrodynamics}

To isolate the effect of diffusive transport from the one of hydrodynamic convective motion on the stability of the binary mixture we first study the stability of stratified films without fluid motion, i.e. we set $w_{1}=0$ in Eq. (26) and only solve (26), (31), (32) and (33) together with the equations for the steady states. This implies as well that the free surface remains 

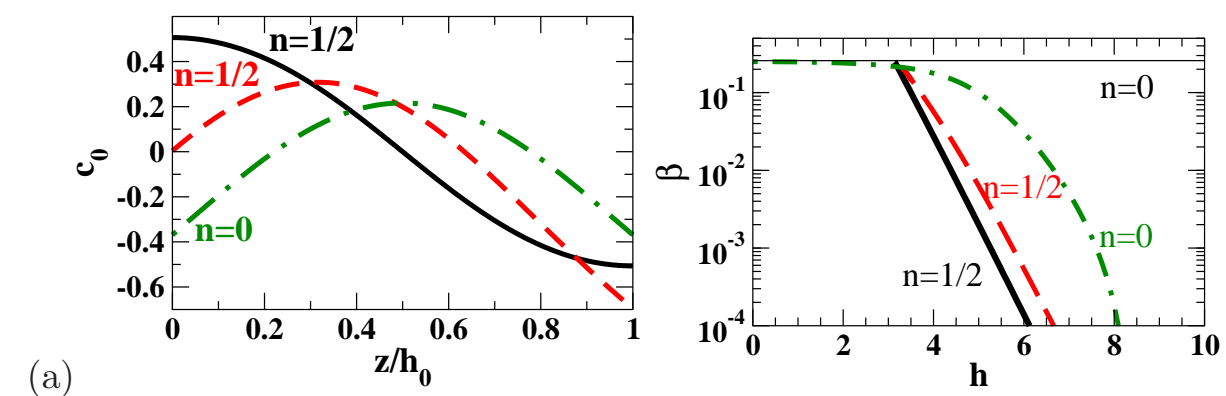

(a)
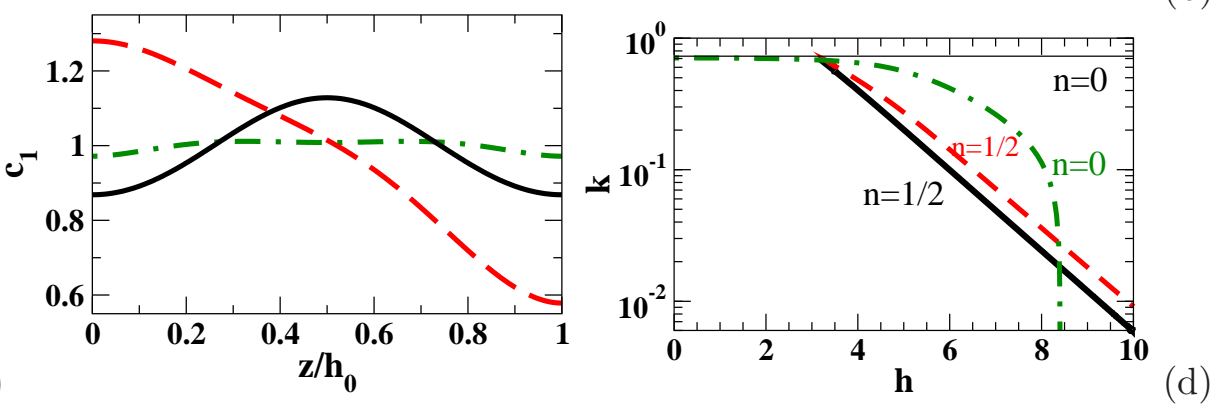

Figure 5: (color online) Characteristics of the horizontal instability modes in the case of purely diffusive transport for neutral $\left(a^{ \pm}=b^{ \pm}=0\right.$, solid lines in (b) and (d)) and symmetrically biased $\left(a^{ \pm}=0.5, b^{ \pm}=0\right.$, dashed and dot-dashed lines in (b) and (d)) surfaces. Panels correspond to (a) concentration profiles $c_{0}(z)$ of the stratified base states at $h_{0}=3.5$ for neutral surfaces $(n=1 / 2$ branch, solid line) and symmetric bias ( $n=0$ branch, dot-dashed line and $n=1 / 2$ branch, dashed line); (c) perturbation profiles $c_{1}(z)$ for $h=3.5$ and line styles as in panel (a); (b) maximum growth rate and $(\mathrm{d})$ associated wavenumber as a function of the film thickness for $n=0$ and $n=1 / 2$ base states (cf. Figs. 1 and 4 of Ref. 37). In all cases $S=1, R e=0, P s / R e=1$.

flat.

\section{Neutral surfaces}

First, we discuss the case of neutral surfaces summarized in Fig. 5. The solid line in panel (a) gives the concentration profile of the base state for neutral surfaces for a film thickness not much above the branching of the $n=1 / 2$ branch from the trivial homogeneous state. The concentration decreases monotonically with vertical position corresponding to a layered film with component 1 enriched near the substrate and component 2 enriched near the free surface (remember that $c=c_{1}-c_{2}$ ). As the film is quite thin, the diffuse interface between the two phases nearly spans all the layer. The solid line in panel (c) gives the perturbation 
mode $c_{1}(z)$.

Figs. 5(b) and (d) show the maximal growth rate and the associated wavenumber, respectively, as a function of the film thickness. Solid lines refer to results for the energetically preferable base state solution. We distinguish two regimes: (i) for $0<h<\pi$ the base state corresponds to the homogeneous film, the most dangerous mode is the horizontal mode discussed above in Section IVA, i.e., the growth rate equals 1/4 and the horizontal wavenumber is constant as well (cf. Fig. 2); and (ii) for $h>\pi$ the energetically preferable base state corresponds to the two-layer film shown in Fig. 5(a) as solid line. It is unstable w.r.t. lateral perturbations, however, the growth rate and wavenumber both decay exponentially with increasing film thickness. The exponential decay indicates that in practical terms a film above $20-50 \mathrm{~nm}$ might appear to be stable w.r.t. lateral concentration perturbations when only diffusive transport is taken into account. The corresponding dispersion relations (not shown) are similar to Fig. 1(a), i.e., $\beta_{\max } \rightarrow 0$ for $k_{\max } \rightarrow 0$. In the neutral case the eigenmodes $c_{1}(z)$ show an up-down symmetry (cf. solid line in Fig. 5 (c)). The mean concentration will develop a horizontal variation as $\int c_{1}(z) d z$ strongly deviates from zero. The variation is strongest along the diffuse interface. The thin solid lines in Figs. 5)(b) and (d) indicate the behavior for the (energetically unfavorable) homogeneous $n=0$ state for $h>\pi$.

\section{Symmetrically biased surfaces}

Results for symmetrically biased surfaces with $a^{ \pm}=0.5$ are given as dashed and dotdashed lines in Fig. 5. The energetical bias strongly influences the base states (see Figs. 3 to 5 of Ref. 37). All branches known from neutral surfaces are modified already for weak bias. Their number reduces with increasing bias via a sequence of bifurcations. For strong bias normally only a 3-layer sandwich film survives.

Even for small thicknesses $h<\pi$ the composition of the base state is not uniform any more, i.e., the homogeneous solutions on the $n=0$ branches are modified and become (weakly) stratified. The corresponding norm $\|\delta h\|$ increases with $h$ and $a^{+}$. The strongly stratified $n=1 / 2$ solution branch bifurcates before from the (modified) $n=0$ branch, however, the bifurcation is shifted from $h=\pi$ to $h \sim 3.3$ (see Fig. 5). The bifurcated $n=1 / 2$ branch is always the energetically favorable one.

The concentration profiles given in Fig. [5(a) (dashed line for $n=1 / 2$ branch and dot- 
dashed line for $n=0$ branch) indicate that the preference for component 2 at the top and at the bottom surface causes component 1 to concentrate within the film giving rise to a local concentration maximum at $z / h_{0} \sim 0.3$ for the $n=1 / 2$ solution and $z / h_{0}=0.5$ for the $n=0$ solution. This corresponds to the creation of a 3-layer sandwich structure similar to the $n=1$ solution for unbiased surfaces. $\underline{37}$

At small thicknesses below $\approx \pi$, the maximal growth rate as well as the associated wavenumber for the $n=0$ branch now decrease slightly with $h$ (Fig. 5(b) and (d)). For larger $h$ growth rate and wavenumber decay faster than exponentially, until for $h \approx 8.3$ the $n=0$ branch gains stability with respect to lateral perturbations. This is, however, practically of no importance as for $h>3.3$ the $n=0$ branch is not the energetically favorable one. The energetically favorable one is the $n=1 / 2$ branch that behaves similar as in the case of neutral surfaces.

The eigenmodes $c_{1}$ are given in Fig. 5 (c). For the $n=1 / 2$ branch the mean concentration $\overline{c_{1}}$ is smaller than in the case of neutral boundaries, i.e., the perturbation is not predominantly lateral. The horizontal variation is smallest at the top and strongest along the bottom (cf. Fig. $5\left(\right.$ (c)). The $n=0$ branch is unstable w.r.t. a nearly purely lateral mode as $c_{1}(z) \approx 1$. The direct comparison of the neutral and the biased case in Fig. 5 shows, however, that the symmetrically biased surfaces actually decrease the lateral instability of the layered $(n=1 / 2)$ branch. Note finally, that the $n=0$ branch is much less unstable for symmetrical bias than for neutral surfaces.

\section{Antisymmetrically and asymmetrically biased surfaces}

Stability results for antisymmetric surfaces with $a^{+}=-a^{-}=0.2$ in the case of purely diffusive transport are presented in Figs. 6(b) and (d) using dashed lines (notice that dashed lines are superposed to solid lines). Panel (a) and (c) present selected base state $\left(c_{0}(z)\right)$ and perturbation $\left(c_{1}(z)\right)$ profiles. The figures give results for the $n=1 / 2$ branch only as it is the energetically favorable one (cf. Figs. 6 to 8 of Ref. 37 ).

The boundary conditions for antisymmetric surfaces favor a bi-layer structure of the film, i.e., they give rise to a monotonous change of $c_{0}(z)$ with $z$ (Fig. 6(a)). Growth rate and wavenumber of the most dangerous lateral mode both decrease with increasing film thickness until the film becomes laterally stable at about $h=4.5$. This implies that an antisymmetric 

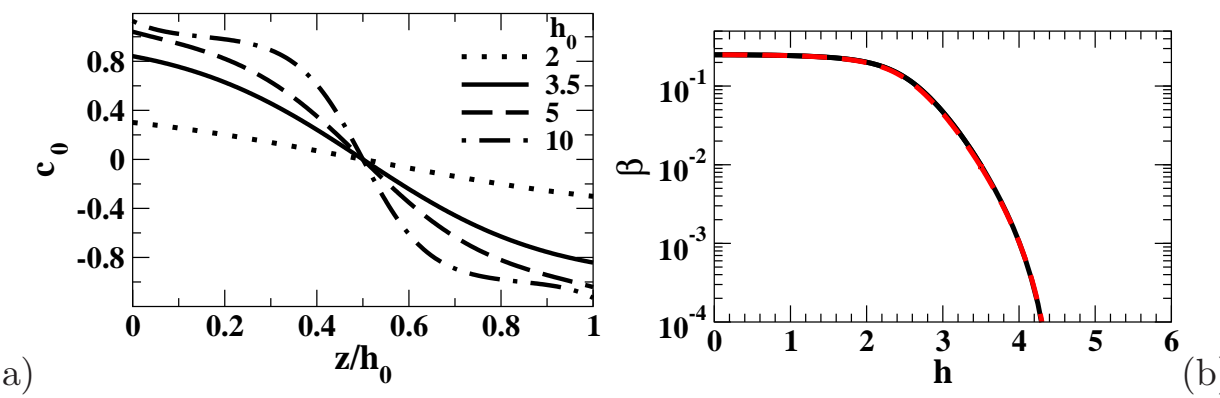

(a)
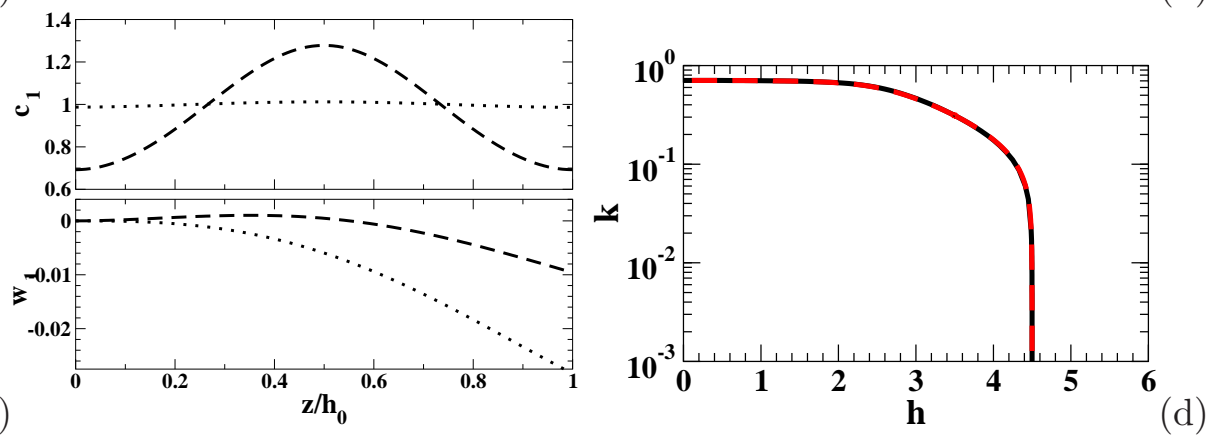

Figure 6: (color online) Characteristics of the horizontal instability modes for antisymmetrically biased surfaces with $a^{+}=0.2, a^{-}=-0.2$ and $b^{ \pm}=0$. Panel (a) gives base state concentration profiles for solutions on the energetically favorable $n=1 / 2$ branch (see Figs. 6 and 8 of Ref. 37 ) for selected film thickness as indicated in the legend. Panel (c) presents the corresponding perturbation modes $c_{1}(z)$ and $w_{1}(z)$ for $h=3.5$. Note that the $c_{1}$ curves with and without convection lie on top of each other. Panels (b) and (d) present characteristics of the horizontal instability modes for purely diffusive transport (dashed lines) and for coupled transport by diffusion and convection (solid lines). Note, that solid and dashed lines coincide for the chosen axis scales. (b) Maximum growth rate and $(\mathrm{d})$ associated wavenumber as a function of the thickness for the branch $n=1 / 2$. Parameters: $\mathrm{S}=1, \mathrm{Re}=0$ and $\mathrm{Ps} / \mathrm{Re}=1$.

bias has a much stronger stabilizing effect than a symmetric one (compare Figs. 5 and 6).

The corresponding dispersion relations (not shown) are similar to Fig. 1(a). The eigenmodes $c_{1}(z)$ for the $n=1 / 2$ stratified film shows an up-down symmetry. It indicates that the concentration will develop a horizontal variation that is strongest along the diffuse interface and weakest at the surfaces (cf. Fig. 6(c)).

The stability behavior for asymmetric surface bias is shown with dashed lines in Fig. 9(b) and $(\mathrm{d})$ for $a^{+}=0.2$ and $a^{-}=0$. The corresponding base state profiles $c_{0}(z)$ and eigenmodes $c_{1}(z)$ are given in Fig. 9(a) and (c), respectively. Only results for the energetically favorable $n=1 / 2$ branch (cf. Figs. 9 and 10 of Ref. 37) are shown. The growth rate and wavenumber 
of the most dangerous mode decrease with increasing thickness $h$ until the film becomes laterally stable at about $h=5.6$. The stabilizing effect is weaker than in the antisymmetric case, but much stronger than in the symmetric one (compare Figs. 5 to 9). Dispersion relations (not shown) are similar to Fig. 1(a).

Before we investigate the influence of convective motion in the next section we summarize our findings for the purely diffusive case: For homogeneous films, we have found that energetically biased surfaces (no linear bias $a^{ \pm}=0$, purely quadratic bias $b^{ \pm} \neq 0$ ) have a strongly stabilizing effect for small thicknesses (with the exception of an antisymmetric bias). The influence of the boundaries becomes weaker for larger film thicknesses. For stratified films, an energy bias at the surfaces (purely linear bias $a^{ \pm} \neq 0$, no quadratic bias $b^{ \pm}=0$ ) stabilizes the layered film against lateral perturbations for asymmetric and antisymmetric biases. In both cases the $n=1 / 2$ stratified films are stable above some critical film thickness. A symmetric bias, however, does only slightly stabilize the layered films as compared to the case of neutral surfaces. For symmetric and neutral surfaces the maximal growth rate decreases exponentially with increasing film thickness, but no critical film thickness was found.

\section{B. Stability with hydrodynamics}

After having studied stability in the case of purely diffusive transport we now allow as well for transport by convection, i.e., we introduce the perturbations of the velocity fields back into the model. Note that the base states are identicl to the ones in the purely diffusive case. However, the possible convective motion of the fluid mixture may alter their lateral stability, and allow for an evolving deflection of the free surface, and, in consequence, lead to films that show lateral modulations of composition and surface profile.

The case of a homogeneous film is discussed above in Section IV: The linear perturbations of the concentration and the velocity fields are decoupled implying that hydrodynamics has no influence on the evolution of the homogeneous film in the linear stage. No surface deflection can occur.

More detailed considerations are needed for vertically stratified films as there the perturbations of concentration and velocity are coupled in the linearized bulk equations and boundary conditions. As above we distinguish the cases of neutral and energetically biased surfaces. For neutral surfaces the vertical gradient of the base state concentration $\left(\left.\partial_{z} c_{0}\right|_{0, h}\right)$ 

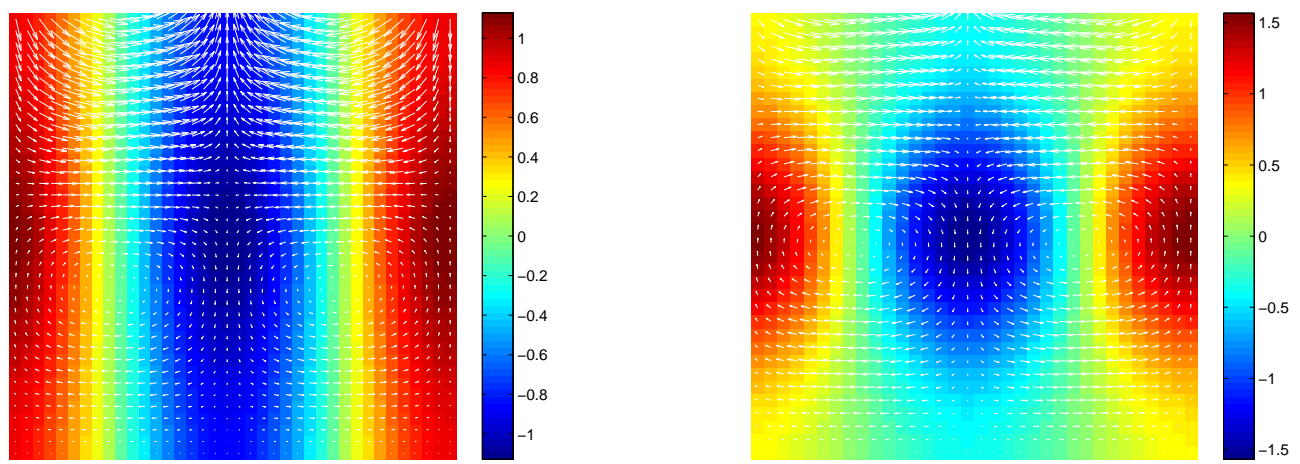

(a)

(b)
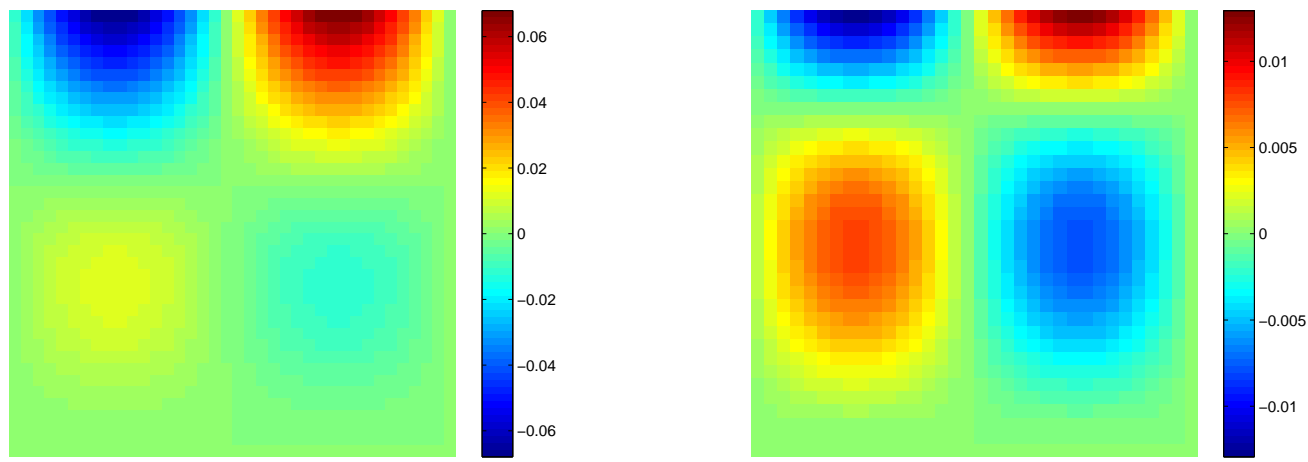

(c)

Figure 7: (color online). Strength of the perturbation fields for neutral surfaces $\left(a^{ \pm}=0\right)$. Panels (a) and (b) give the concentration field $c_{1}(x, z)$ with a superposed vectorial representation of the velocity field $\left(v_{1}, w_{1}\right)$ (white arrows). Panels (c) and (d) give the corresponding stream functions $\psi_{1}(x, z)$. The film thickness is (a,c) $h=3.5$ and (b,d) $h=5$. The color bars give the corresponding field 'strength'. Remaining parameters are $\mathrm{S}=1, \mathrm{Re}=0$ and $\mathrm{Ps} / \mathrm{Re}=1$. Horizontal wave numbers are $k=0.558(h=3.5)$ and $k=0.202(h=5)$. The lateral and vertical size of each image correspond to the lateral period $2 \pi / k$ and the film thickness $h$, respectively.

is zero at both surfaces and increases or decreases into the bulk. This implies that the flow has to be driven by the internal diffuse interface between the two components [cf. momentum equation Eq. (1)]. Corresponding flow and concentration perturbation fields are illustrated for different film thicknesses in Fig. 7. We will call this type of forcing "bulk Korteweg driving".

For energetically biased surfaces the $\left.\partial_{z} c_{0}\right|_{0, h}$ are not zero, they might even have their extrema at the surfaces [cf. Fig. 5(a)]. This implies that the driving Korteweg forces can be localized near the surfaces. Liquid motion, however, is suppressed at the solid substrate 

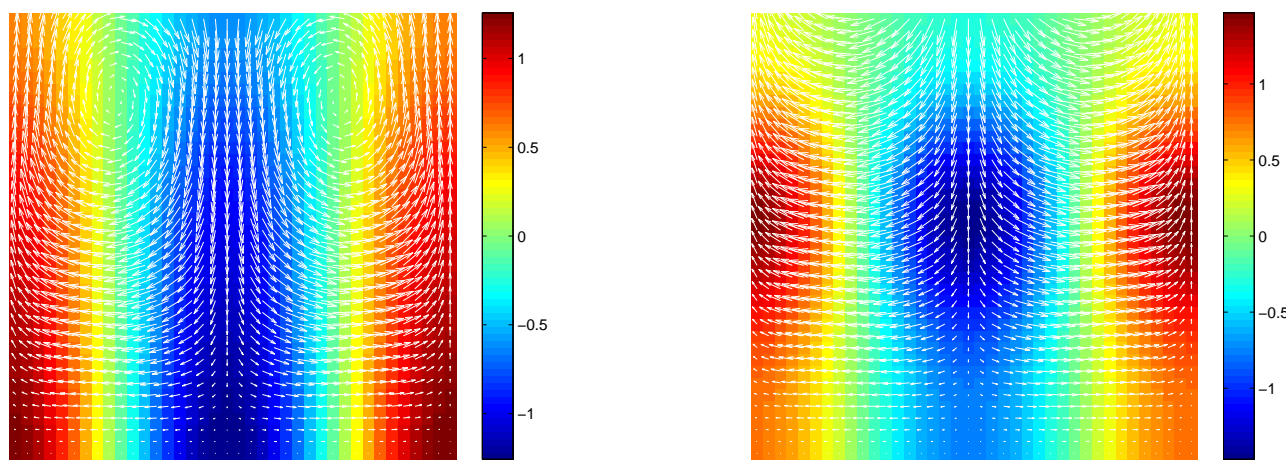

(a)

(b)
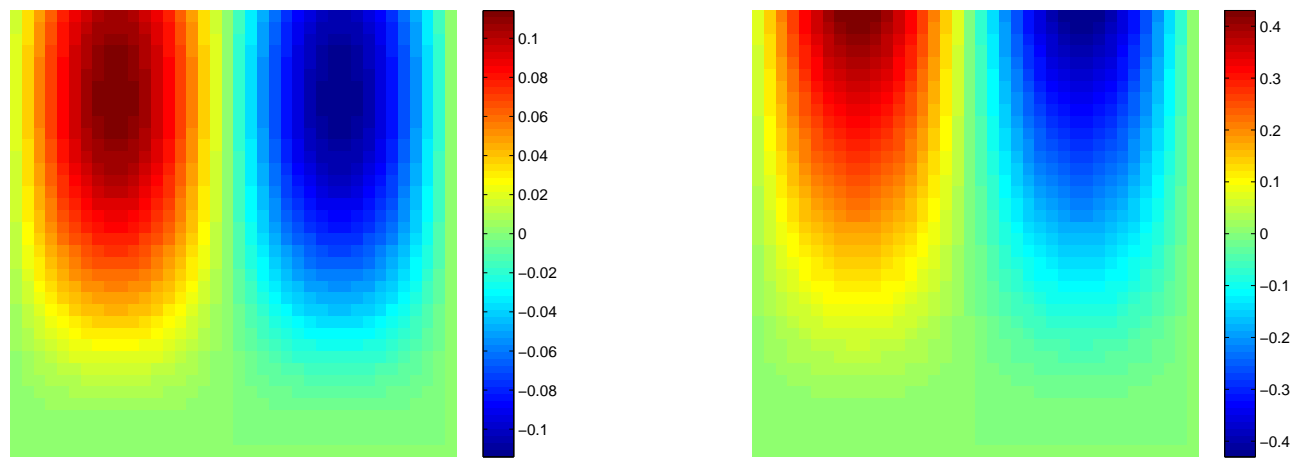

(c)

Figure 8: (color online). Presented are the perturbation fields for symmetric surface bias $\left(a^{ \pm}=0.5\right)$. Panels (a) and (b) give the concentration field $c_{1}(x, z)$ with a superposed vectorial representation of the velocity field $\left(v_{1}, w_{1}\right)$ (white arrows). Panels $(\mathrm{c})$ and $(\mathrm{d})$ give the corresponding stream functions $\psi_{1}(x, z)$. The film thickness is $(\mathrm{a}, \mathrm{c}) h=3.5$ and $(\mathrm{b}, \mathrm{d}) h=5$. The color bars give the corresponding field 'strength'. Remaining parameters are $\mathrm{S}=1, \mathrm{Re}=0$ and $\mathrm{Ps} / \mathrm{Re}=1$. Horizontal wave numbers are $k=0.594(h=3.5)$ and $k=0.213(h=5)$. Both solutions are on the $n=1 / 2^{b}$ branch. The lateral and vertical size of each image correspond to the lateral period $2 \pi / k$ and the film thickness $h$, respectively.

due to the no-slip condition, but can be strong at the free surface. Corresponding flow and concentration perturbation fields are illustrated for different film thicknesses in Fig. 8. This "surface Korteweg driving" corresponds to the classical Marangoni driving at a free surface for one-component systems.

Note, that strictly speaking the flow is only driven by the Korteweg term in the bulk equations due to the exact cancellation of the Marangoni and Korteweg terms in the tangential stress boundary condition for the perturbations. As one is, however, still able to distinguish 

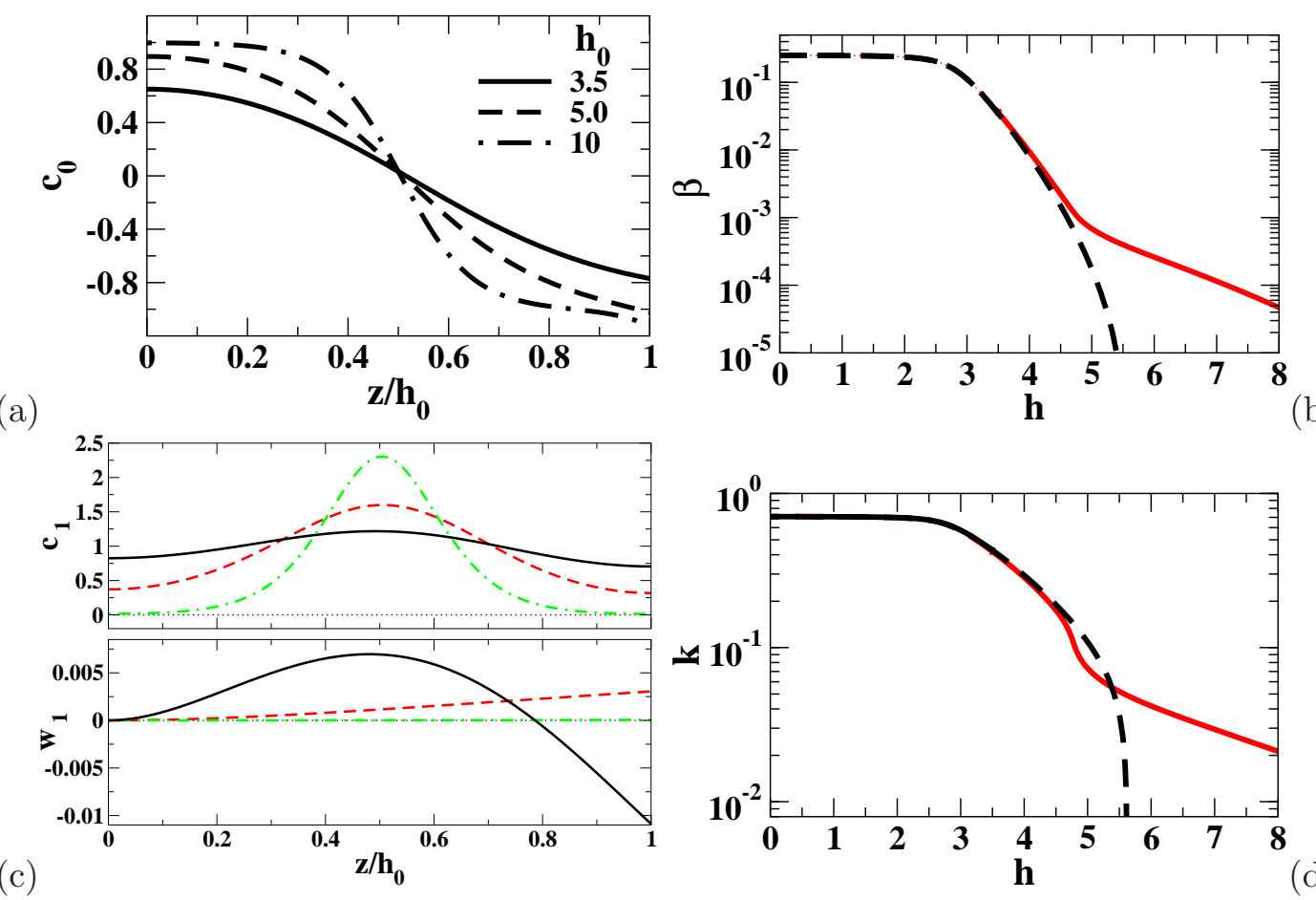

Figure 9: (color online) Results for asymmetrically biased surfaces with $a^{+}=0.2$ and $a^{-}=b^{ \pm}=0$. Panel (a) gives base state concentration profiles on the energetically favorable $n=1 / 2$ branch (see Figs. 9 and 10 of Ref. 37) for selected film thickness as indicated in the legend. Panel (c) presents the corresponding perturbation modes $c_{1}(z)$ and $w_{1}$. Note that the $c_{1}$ curves with and without convection can not be distinguished by eye. Panels (b) and (d) present for for this $n=1 / 2$ branch the maximal growth rate and the associated wavenumber, respectively, of the horizontal instability modes for purely diffusive transport (dashed lines) and for coupled transport by diffusion and convection (solid lines). The remaining parameters are $\mathrm{S}=1, \mathrm{Re}=0$ and $\mathrm{Ps} / \mathrm{Re}=1$.

a driving at the free surface and a driving at the diffuse interface we call the former either "surface Korteweg driving" or "Marangoni driving" and the latter "bulk Korteweg driving". In the following we discuss the individual cases in more detail.

\section{Neutral surfaces}

The Marangoni number is expressed as $\mathrm{Ma}=a^{+} \mathrm{S}=a^{+} \gamma_{0} / l E$. Therefore an upper neutral surface $\left(a^{+}=0\right)$ means that gradients of concentration along the free surface do not give rise to gradients of surface tension, i.e., do not drive a flow. Fig. 10 shows the maximum growth 


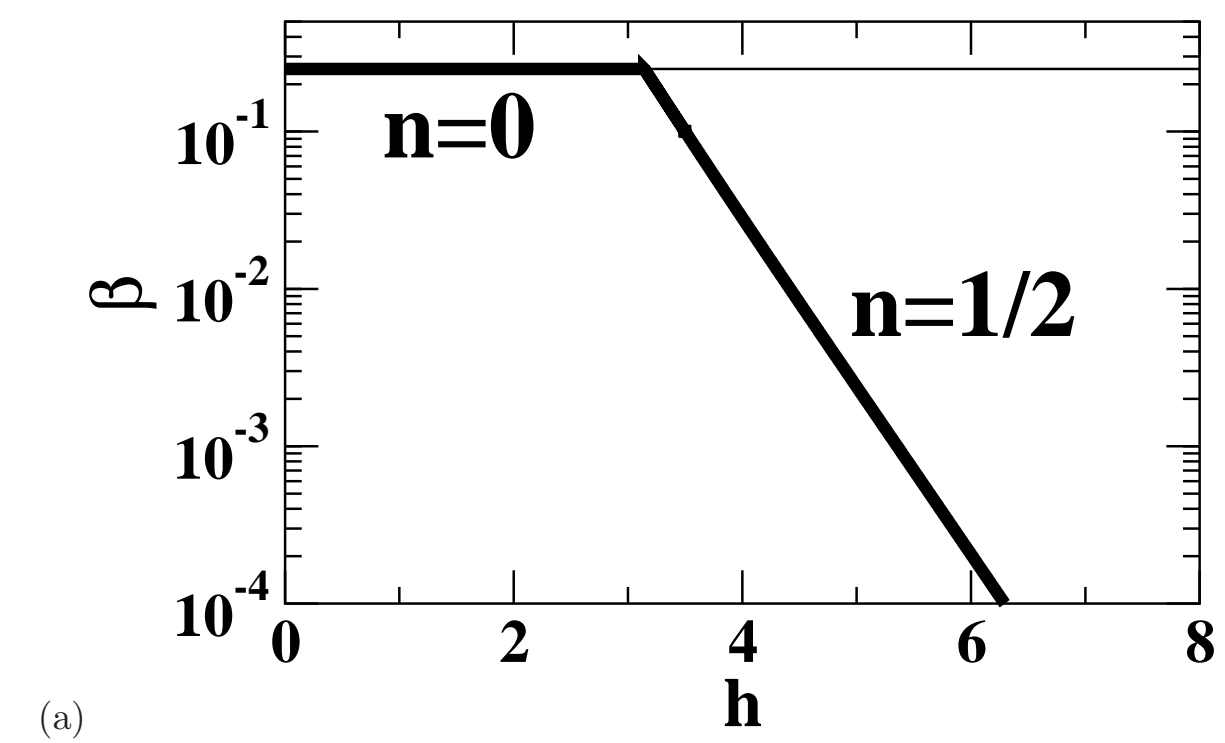

(a)

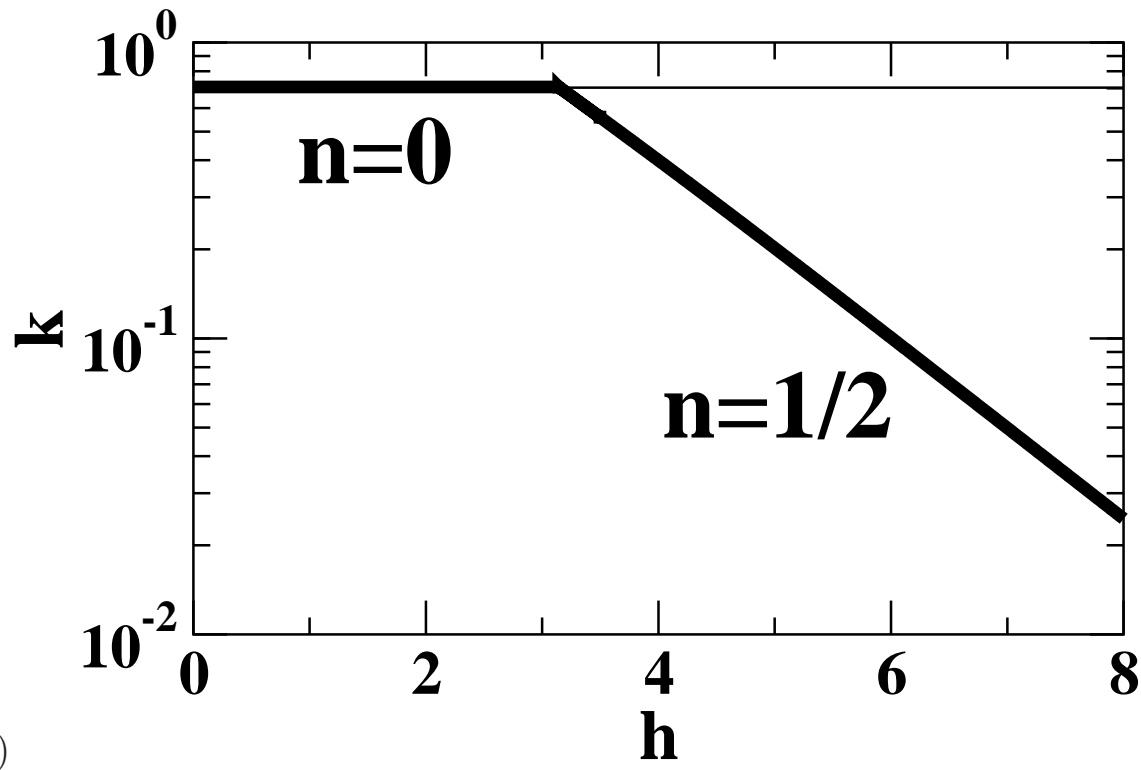

Figure 10: Characteristics of the horizontal instability modes in the case of coupled transport by diffusion and convection for neutral surfaces $\left(a^{ \pm}=b^{ \pm}=0\right)$. (a) Maximum growth rate, and (b) associated wavenumber as a function of the film thickness for $n=0$ and $n=1 / 2$ base states (see Figs. 1 and 4 of Ref. 37). Remaining parameters are $S=1, R e=0$ and $P s / R e=1$.

rate and associated wavenumber as a function of the film thickness. A first comparison to Fig. 5 seems to indicates that convective transport does only cause small changes. However, a careful analysis shows that the instability is accelerated by up to $\sim 50 \%$ (as measured at $h=6$ : purely diffusive $\beta=1.4 \times 10^{-4}$; with convection $\beta=2.1 \times 10^{-4}$ ). This is difficult to discern in the figures as the growth rate decreases exponentially with increasing film thickness. 
The effect of convective transport on the growth rate is, however, a result of a rather dramatic change of the velocity field inside the film. By definition there is no such field in the purely diffusive case, whereas now with the incorporation of convective transport 'convection rolls' appear driven by the bulk Korteweg term in Eq. (27). This new dynamics inside the film allows for a surface deflection to evolve. Figs. 7( $(c, d)$ illustrate the convective motion by showing the stream function corresponding to the perturbation velocity field $\left(v_{1}, w_{1}\right)$ in a vertical cut through the film for (c) $h=3.5$ and (d) $h=5$. In the latter case one can clearly appreciate how the convective cells are driven from within the bulk. At each lateral position there is a well visible upper cell and a less visible lower cell that rotate in opposite direction. For $h=3.5$ the lower cell is very weak as it is strongly 'damped' by the no-slip condition at the substrate. The strength of the vertical flow at the upper surface is proportional to the evolving local surface deflection. Figs. 7(a,b) give the corresponding perturbation fields for the concentration $c_{1}$ and as well indicate the velocity field as superposed white arrows. For $h=3.5$ the field $c_{1}$ shows nearly vertical stripes, i.e., it is almost a purely horizontal mode. For $h=5$ the lateral modulation in the concentration field is less developed at the surfaces. It is strongest in the region of the diffuse interface.

\section{Symmetrically biased surfaces}

Fig. 11 gives the main stability result for symmetrical bias (solid lines) with $a^{ \pm}=0.5$, and $\mathrm{S}=1$, therefore $\mathrm{Ma}=0.5$ in comparison to the case of purely diffusive transport (dashed lines). There are two important differences: (i) Up to $h \approx 6$ the $n=0$ branch is with convection only slightly more unstable. In contrast, for $h>6$ the decrease in the growth rate slows strongly down, i.e., with further increasing thickness the branch becomes orders of magnitude more unstable than without convection. This is, however, only of secondary importance as we expect the branch to be experimentally not of great importance. It represents the energetically favorable solution only below $h \approx 3.3$. The behavior is nevertheless interesting and we will later on encounter similar results for relevant branches.

(ii) Remarkable is the splitting in two of the $n=1 / 2$ branch. It is a consequence of the breaking of the up-down symmetry by the hydrodynamic boundary conditions (noslip vs. free surface). Symmetries and resulting multiplicities of branches of base states are discussed in 37 . In particular, for symmetric bias the $n=1 / 2$ branch represents two solutions 


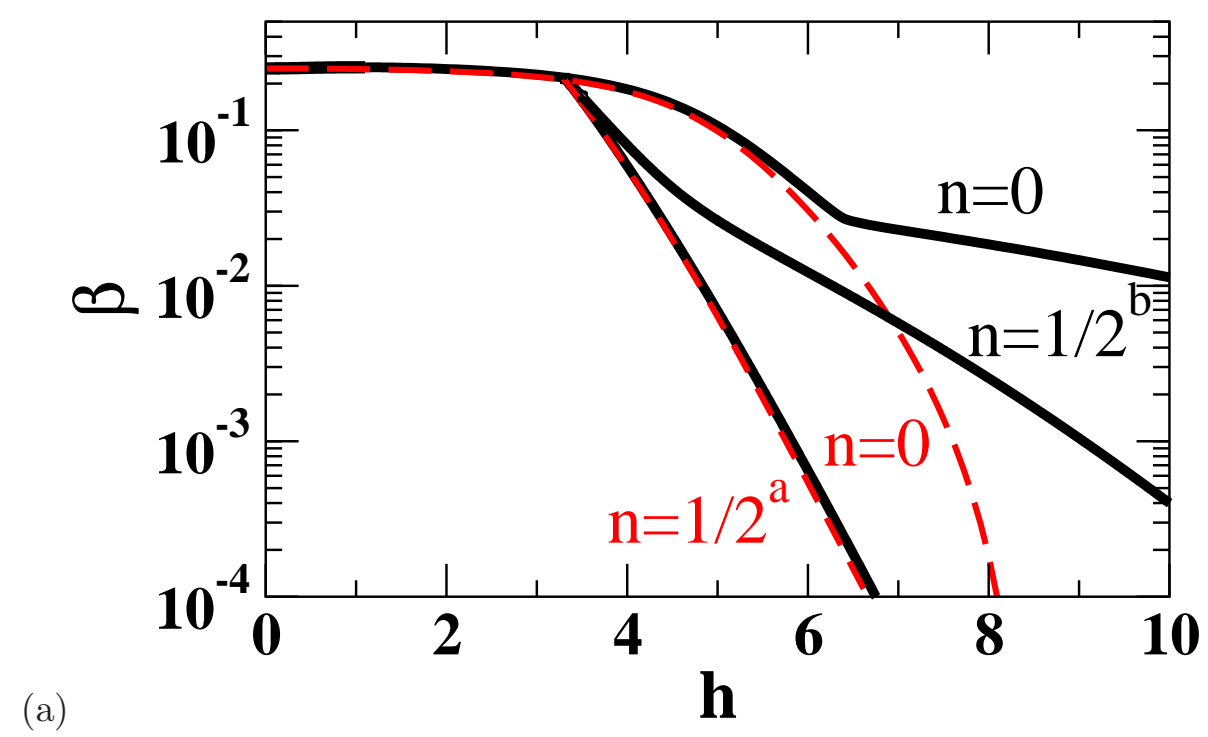

(a)

(b)

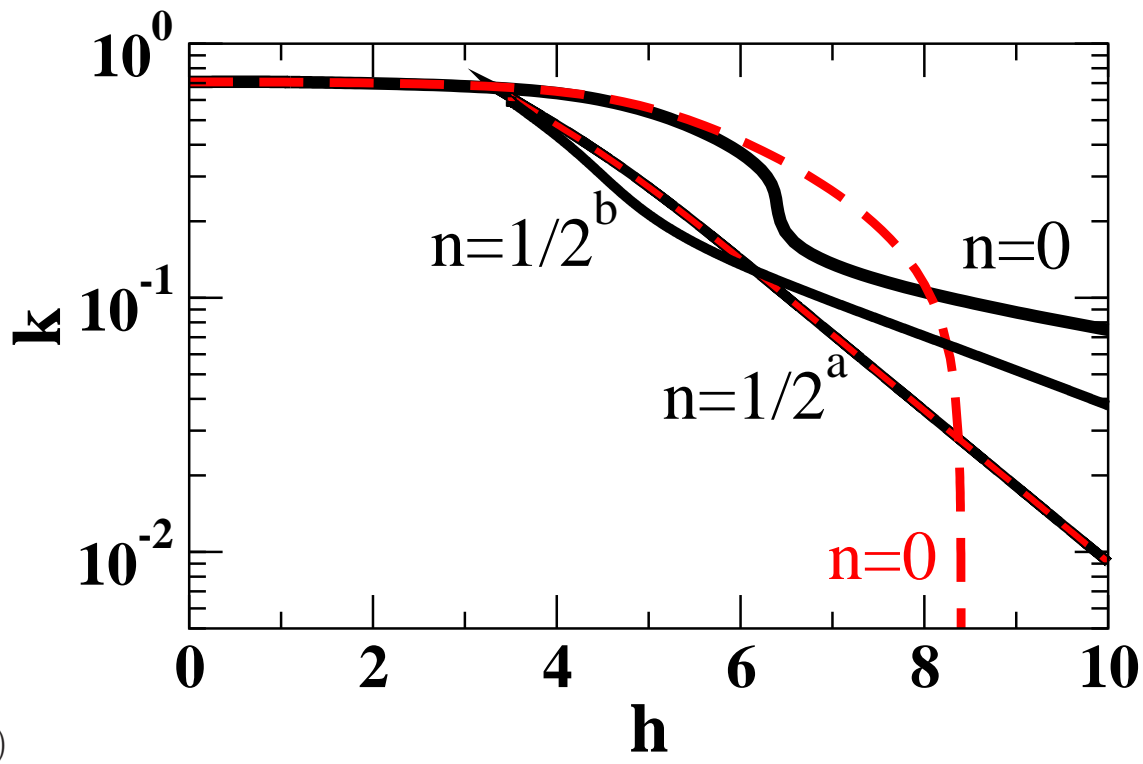

Figure 11: (color online) Characteristics of the horizontal instability modes for purely diffusive transport (Dashed lines) and for coupled transport by diffusion and convection (solid lines). Surfaces are symmetrically biased with $a^{ \pm}=0.5$. Panel (a) shows the maximal growth rate and (b) the associated wavenumber as a function of the thickness for $n=0$ and $n=1 / 2$ base states (see Figs. 3 and 4 of Ref. 37). Remaining parameters are $S=1, R e=0$ and $P s / R e=1$.

related by the symmetry $c_{0}(z) \rightarrow-c_{0}(h / 2-z)$. The two solutions behave identically when increasing the energy bias. Without hydrodynamics they have as well identical stability properties. However, as convective transport is included the symmetry is broken and the two solutions acquire different stability properties (marked as branches $1 / 2^{a}$ and $1 / 2^{b}$ in Fig. 11). Both branches are more unstable with convection than without, however, whereas 
for the branch $n=1 / 2^{b}$ the difference reaches one order of magnitude already at $h \approx 5$, the branch $n=1 / 2^{a}$ is only slightly more unstable.

The velocity field inside the film is quite different from the one for neutral surfaces given above in Fig. 7 For symmetrical bias the surface tension depends on concentration and a Marangoni driving at the free surface is possible. Remember, however, that the 'classical' solutal Marangoni effect manifests itself in the linear analysis through a surface Korteweg driving active in a diffuse region in the vicinity of the sharp free surface. The corresponding perturbations of concentration and velocity fields are illustrated in Fig. 8 for the $n=1 / 2^{b}$ branch at $h=3.5$ (left) and $h=5$ (right). In the representation of the velocity field as stream function $\psi_{1}(x, z)$ one can clearly discern 'convection rolls' driven by the free surface, i.e., at each lateral position there is only one convection cell that is concentrated near the free surface. As the driving occurs where the film is hydrodynamically most mobile the destabilizing effect is much stronger when caused by the Marangoni mode (surface Korteweg mode) than when caused by the bulk Korteweg mode. In consequence, for larger thickness the roll reaches less deeply into the layer. Figs. $8(a, b)$ give the corresponding perturbation fields for the concentration $c_{1}$ and as well indicate the velocity field as arrows. For $h=3.5$ the field $c_{1}$ shows again a rather horizontal mode. It has, however, a stronger vertical element than in the neutral case above. Here, the lateral modulation is strongest at the substrate and weakest at the free surface. For $h=5$ there is nearly no lateral modulation at the free surface. The modulation is strongest around the diffuse interface and is less developed at the substrate.

As in the neutral case the convection allows for a surface deflection to evolve. Fig. 12)(a) shows - using $h=3.5$ as example - the relative strength of the surface deflection $h_{1}=$ $w_{1} / \beta$ as a function of the lateral wavenumber where $w_{1}$ is the value at the vertical velocity component at the free surface. Fig. 12(a) gives as well the corresponding dispersion relation $\beta(k)$ to facilitate the identification of the physically most relevant value of $h_{1}$ at the maximal growth rate. Inspecting the full range of $k$ one finds that the maximal deflection occurs at small but finite $k$. From the maximum value $h_{1}$ decreases monotonically until reaching negative values at large enough $k$. Note that the mode of maximal deflection does not correspond to the most dangerous mode. We add two remarks regarding the interpretation of the $h_{1}(k)$ curve: (i) The fact that $h_{1}(k=0) \neq 0$ does not contradict material conservation as the $k=0$ mode is marginally stable $(\beta=0)$ and not unstable. (ii) The deflection amplitude 


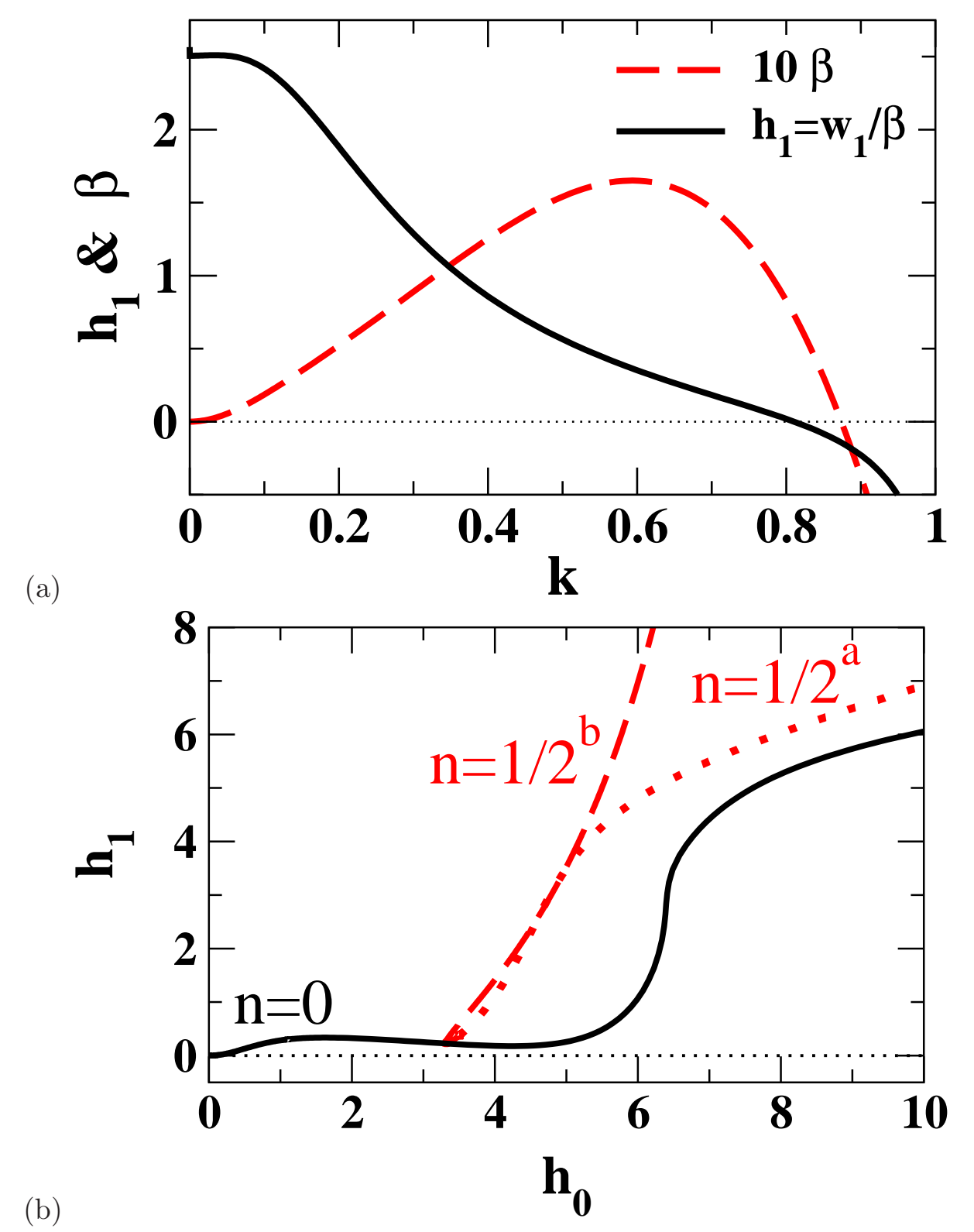

Figure 12: (color online) Panel (a) shows the relative strength of the surface deflection $h_{1}=w_{1} / \beta$ (solid line) and growth rate (dispersion relation, dashed line) as a function of the wavenumber for a fixed thickness $h=3.5$ for the branch $n=1 / 2^{b}$. (b) The deflection $h_{1}$ at the maximum of the dispersion relation is given as a function of the film thickness for the $n=0$ branch (solid line) and the branches $n=1 / 2^{a}$ (dotted line) and $n=1 / 2^{b}$ (dashed line) for symmetrically biased surfaces with $a^{ \pm}=0.5$ and $b^{ \pm}=0$. The remaining parameters are $\mathrm{S}=1, \mathrm{Re}=0, \mathrm{Ps} / \mathrm{Re}=1$. 
$h_{1}$ does not correspond to an absolute amplitude value, it rather represents the relative importance and the relative sign of the surface deflection as compared to the perturbation of the concentration field (cf. Section III).

The accompanying Fig. 12(b) presents the dependence of the surface deflection of the most dangerous linear mode on film thickness for all branches discussed above. For the $n=0$ branch in the range below $h \approx 3.3$, where it is energetically favorable the deflection first increases from zero to $\approx 0.35$, then decreases again. Above $h \approx 3.3, h_{1}$ continues to decrease till $\approx 0.17$ at $h \approx 4.2$ before increasing strongly at about $h \approx 6$ in accordance with

the change in the $\beta_{\max }(h)$ dependence discussed in connection with Fig. 11, For the strongly stratified $n=1 / 2$ branches the deflection increases quickly with film thickness above their bifurcation from the $n=0$ branch indicating the importance of the surface deflection for the lateral instability modes. All these findings strengthen the above conclusion that at small thicknesses diffusion dominates even if convection is possible. Then there occurs a rather sharp transition over a well defined $h$-range (for the present parameter set at about $h=6$ ) where the convective influence becomes important.

\section{Antisymmetrically and asymmetrically biased surfaces}

Results for antisymmetrical bias with $a^{+}=-a^{-}=0.2$ are included above in Fig. 6 for the energetically preferred branch $n=1 / 2$. Contrary to symmetrical bias, one finds that cases without and with convection are nearly identical. For instance, at $h=3.5$ the growth rate with convection is only two per cent larger than without hydrodynamics and the $c_{1}$ perturbation fields can visually not be distinguished (cf. Fig. 6(c)). In both cases the films stabilize w.r.t. lateral instability modes at $h \sim 4.5$. The following consideration might, however, help to understand the finding: The film stabilizes at about $h=4.5$. Below this rather small thickness the results with and without convection are neither well distinguished in the other studied cases. That means the antisymmetrical bias exercises such a strong stabilizing influence that films are already stable 'before' (in terms of film thickness) convective motion becomes relevant. The velocity field $w_{1}$ (cf. Fig. 6(c)) changes non-monotonously with $z$ indicating a pair of convection rolls as in Fig. 7 . This indicates that the weak convection is driven from the diffuse interface within the film.

Finally, we consider the case of asymmetrical bias. Fig. 9 above gives results for the 
energetically favorable $n=1 / 2$ branch at $a^{+}=0.2$ and $a^{-}=0$ in comparison to the purely diffusive case. The overall behavior resembles strongly the one described for symmetrical bias above in Section VB2 for the influence of convection on the $n=0$ branch: up to $h \approx 4.5$ the profile is only slightly more unstable with convection than without. In contrast, for $h>4.5$ the film is much more unstable with convection. Without convective motion the film stabilizes at $h_{c} \approx 5.8$. Allowing for convection and therefore for the evolution of a surface deflection the film remains laterally unstable above $h_{c}$. The growth rate and wavenumber of the most unstable mode still decrease roughly exponentially with increasing film thickness.

The rationale behind this finding is that convective motion does not only add a second transport process to the dynamics but it as well allows the film to realize a different class of solutions, namely, films with laterally modulated concentration and thickness profiles. This qualitative change is as well reflected in a change of the character of the perturbation velocity profile (cf. Fig. 9(c)). At $h=3.5$ it shows non-monotonous behavior indicating driving from the diffuse interface inside the film whereas at larger thicknesses (shown for $h=5$ ) driving comes from the free surface. This corresponds to the transition from bulk Korteweg driving with perturbation fields that resemble Fig. 7 to surface Korteweg (or Marangoni) driving with perturbation fields that resemble Fig. 8. The relative strength of the surface deflection $h_{1}$ (not shown) shows similar behaviour as for the $n=0$ branch in the symmetric case (see solid line in Fig. 12(b)).

We conclude this section with a remark on the character of the dispersion relation. For larger film thicknesses we find wavenumber ranges corresponding to complex modes. Although they seem to be of minor importance (as we find the most dangerous mode to be always real) they are interesting from a theoretical point of view. They seem to be closely related to the possibility of surface deflection, however, their physical interpretation remains elusive. They appear in parameter ranges where several existing real modes re-connect in a different way. However, we carefully checked that they are no numerical artifact: they do not depend on details of the used discretization and are reproducible over several orders of magnitude of numerical tolerances in the employed continuation procedure. Open questions related to this point will be the subject of further investigations. 

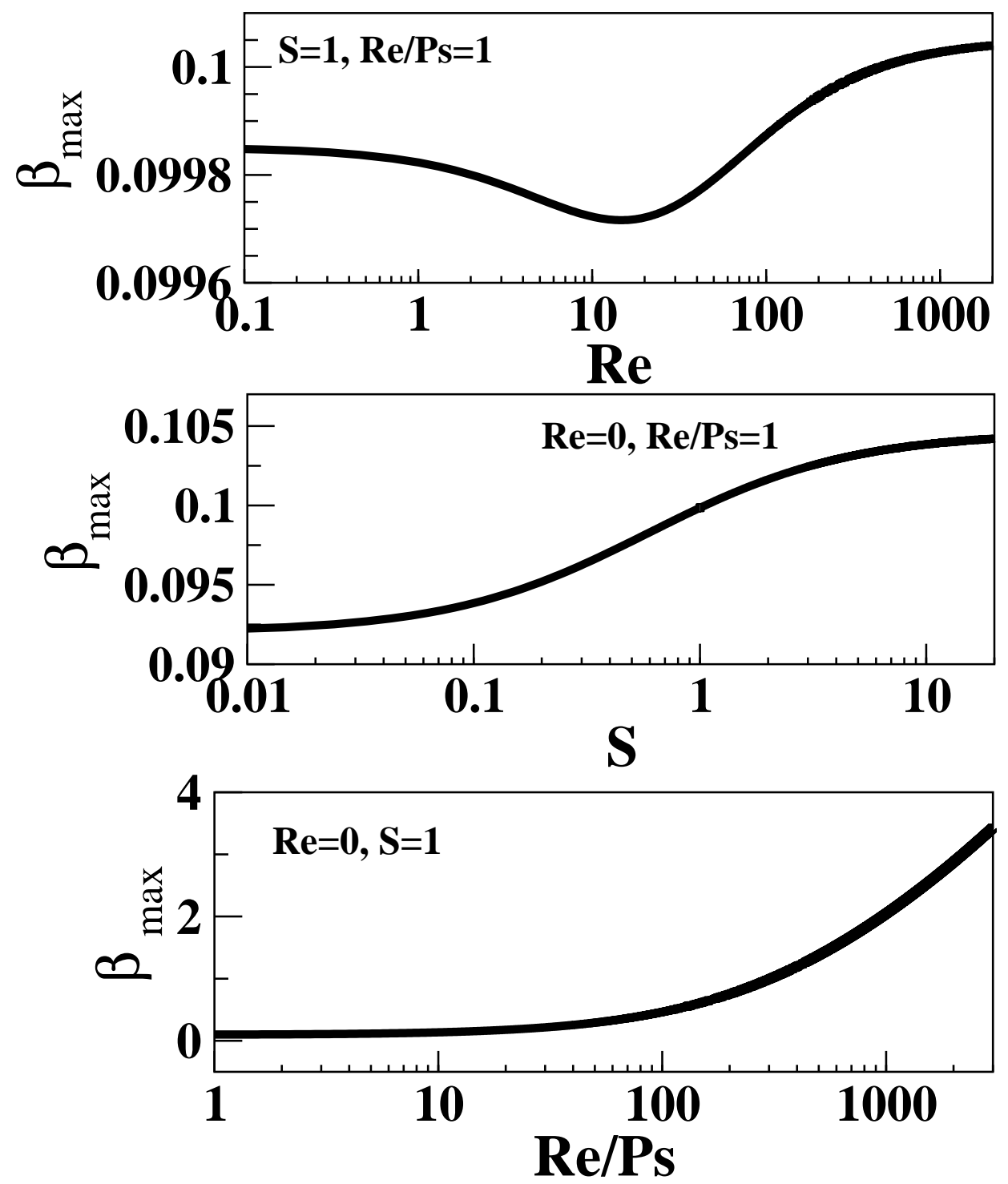

Figure 13: Dependence of the maximal growth rate as a function of (a) the Reynolds number Re, (b) the surface number S, and (c) the ratio Re/Ps. The curves are obtained for a stratified film of the branch $n=1 / 2$ with $h=3.5$ and neutral surfaces $a^{ \pm}=b^{ \pm}=0$.

C. Influence of Re, Re/Ps and S

One can expect that for different types of binary mixtures such as n-pentane/n-decane mixtures, polystyrene/cyclohexane mixtures, or ionic binary mixtures such as triethyl nhexyl borate in diphenyl ether the parameters that we have kept fixed up to here, e.g., the Reynolds number Re, the ratio Re/Ps, and the dimensionless surface tension S will strongly differ. A full scale study of the influence of those parameters is beyond the scope of the 
present work. However, we would like to indicate the tendencies that are to be expected by looking at one particular example. We show in Fig. 13(a) to (c) the influence of changing $\mathrm{Re}, \mathrm{S}$ and Re/Ps, respectively, on the growth rate of the most dangerous lateral instability mode for the case of neutral surfaces and a film thickness of $h=3.5$. Wavenumbers are only discussed in the text.

When changing the Reynold number (Fig. 13)(a); keeping the other parameters including $\mathrm{Re} / \mathrm{Ps}$ fixed), the growth rate at first (for $\operatorname{Re}<14.5$ ) slightly decreases before increasing until it saturates at about $\mathrm{Re}=1000$. The corresponding wavenumber increases monotonically with $R e$ and also saturates for $R e \simeq 1000$. Note, however, that the differences between the minimal and maximal value of the growth rate and the wavenumber in the studied Re range are only about $0.3 \%$ and $0.5 \%$, respectively. Therefore, in the present scaling and the studied case the variation of the Reynolds number alone has practically no influence on the stability of a mixture with unbiased surfaces. Remember, that the same velocity enters the definition of Re and the time scale that enters $\beta$. Fixing Re/Ps furthermore implies that the ratio of diffusive and convective transport remains constant.

When increasing the surface tension number (Fig. 13(b)) the growth rate and the wavenumber both increase monotonically. The growth rate saturates at about $S \simeq 5$ and the maximum value of the growth rate and wavenumber are close to the ones found for high Re. Although the increase of $\mathrm{S}$ has a slightly larger impact than the increase of Re, the overall variation is still quite small: growth rate $\approx 10 \%$ and wavenumber $\approx 3 \%$ ).

The ratio Re/Ps has a much larger impact on the stability of the mixture (c.f. Fig. 13)(c)): The increase of the growth rate is orders of magnitude larger than when increasing Re or $\mathrm{S}$. The growth rate increases monotonously with increasing Re/Ps, whereas the associate wavenumber decreases. As discussed above Re/Ps corresponds to the ratio of the typical velocity of the viscose flow driven by Korteweg stresses and the typical velocity of diffusive processes. Therefore, an increase of Re/Ps shifts the transition towards a larger influence of convective transport to smaller film thicknesses and results in the behavior presented in Fig. 13)(c). 


\section{CONCLUSIONS}

We have studied the linear stability with respect to lateral perturbations of homogeneous and layered films of polymer mixtures that have a free deformable surface and are bound on the other side by a rigid solid substrate. The paper represents the second part of a series of works that develops and applies a version of model-H suitable for the study of confined systems involving free surfaces.

In the first part (Ref. 37) we had derived a generalized model-H coupling transport equations for momentum, density of one component of the mixture and entropy. The model was then simplified for isothermal systems. Furthermore, we had modeled an energetic bias towards one of the components at the surfaces, had discussed how to include the evolving free surface and had finally determined stratified film base states for various types of energetic bias at the surfaces.

The present second part has focused on the determination of the linear stability properties of the stratified film states studied in Ref. 37. To this aim, we have linearized the governing equations with respect to small perturbations in the velocity and concentration fields, and the surface profile. We have emphasized that hydrodynamic flow has to be accounted for even in the case of extremely slow creeping flow as otherwise surface deflections can not evolve. The resulting linear system of equations and boundary conditions plays a similar role as the coupled Orr-Sommerfeld-type equations for velocity and temperature perturbations describing the linear surface-tension driven instability of a horizontal liquid layer with a deformable free-surface of a heated layer of simple liquid $\underline{68}$.

We have performed a numerical analysis of the stability with respect to lateral modes of homogeneous and stratified base states (quiescent films) for several different cases of energetic bias at the surfaces, corresponding to linear and quadratic solutal Marangoni effects. In passing we have elucidated the close relation between surface Korteweg driving (forcing by Korteweg stresses in the bulk momentum equation for the diffuse layer adjacent to the free surface) and Marangoni driving. The numerical analysis has been performed to a high precision relying on continuation techniques. The latter have, in particular, allowed to follow the most dangerous linear mode over a wide range of a number of important parameters.

For neutral surfaces two types of steady film solutions exist: homogeneous and stratified 
ones. For homogeneous films, perturbations of the composition and velocity fields are linearly decoupled. In consequence, the stability of the films is ruled by the linear modes of a CahnHilliard equation in a confined slab-type geometry. We have found that the film is laterally unstable for all thicknesses in agreement with literature. $\frac{67}{69}$ For layered films, the films become exponentially less unstable with increasing thickness in the cases without and with inclusion of convective transport. In the latter case the decrease is slightly slower, i.e., hydrodynamics further destabilizes the films. We have found that for neutral surfaces and critical mixtures the convective motion is driven by a bulk Korteweg effect, i.e., a forcing through the Korteweg stresses in the diffuse interface region between the two components of the mixture.

Homogeneous films are still a base state for quadratically energetically biased surfaces. We have investigated the case as a benchmark for comparison with literature ${ }^{38,67,69}$. As expected we have found that both - symmetrical and antisymmetrical energetic bias - stabilize the lateral instability below respective threshold thicknesses. This is, however, not the case for an asymmetrical bias; a question that needs further investigation.

We have found that the stability behavior of layered films under different types (symmetrical, antisymmetrical and asymmetrical) of energetic bias is rather rich. For purely diffusive transport, depending on the type of branch and type of bias an increase in film thickness either (i) exponentially decreases the lateral instability or (ii) it entirely stabilizes the film when reaching a threshold thickness. Including convective transport leads in many cases to a small or strong destabilization as compared to the purely diffusive case without changing the behavior qualitatively. Most remarkably, however, in some cases the inclusion of convective transport and the related widening of available configuration space for the film (it may then change its profile) changes the stability qualitatively. For instance, an asymmetrical energy bias results in a stabilization at a threshold thickness when no convection is allowed. With convection the film remains unstable well above this threshold, although it still becomes exponentially more stable with increasing film thickness.

Finally, we have presented results regarding the dependence of the instability on several other parameters, namely, the Reynolds number, the Surface tension number and the ratio of typical velocities of convective transport driven by Korteweg stresses and by diffusive transport. We have identified the latter parameter as the most influential one.

Note that we have entirely focused on the case of a critical mixture, i.e., the case of 
zero mean concentration. A full scale analysis for off-critical systems, for instance, of the dependence of stability on mean concentration has been outside the scope of the present paper and will be pursued elsewhere.

In general, our results have shown that the possibility of a change in the height profile of the fill alters the stability behavior of a film of a binary mixture not only quantitatively but as well qualitatively. Therefore such a possibility has always to be taken into account.

Although, the performed stability analysis yields results regarding stability thresholds, and time- and length-scales of the most dangerous modes, it does not allow to predict the nonlinear short- and long-time evolution of an unstable film of a binary mixture. Results obtained on related two-layer films using long-wave sharp interface models $\underline{70}-\underline{72}$ show that the nonlinear behavior may be quite unexpected. An example are the morphological transitions that may occur in the process of coarsening 71,72 . To fully account for the behavior the free surface model-H should be used to study the time evolution of films of binary mixtures in the nonlinear regime. An alternative approach could focus solely on the final stable structures, i.e., steady state films that are characterized by a steady non-flat surface profiles and internal non-homogeneous concentration profiles.

Finally, we would like to point out that the given analysis only presents a first step towards a fully operative thin film model that is able to describe in a quantitatively correct way the coupled dewetting and decomposition observed in many experiments using films of polymer blends of thicknesses well below 100nm. 56 . A basic ingredient of thin film physics - the effective molecular interactions between film and substrate - has not yet been taken into account. The approach followed in thin film models of simple liquids is to include those interactions as an additional pressure term that depends on film thickness and models wettability. $\underline{1}, \underline{6}$.

Nevertheless, the present theory allows to formulate a hypothesis that may explain why rather thick films (100-200nm) of polymer blends can rupture rather fast: $\underline{73}$ The presently investigated decomposition-caused convection-mediated lateral instability leads to a growing lateral modulation of the film surface. This allows to 'bypass' the rather slow linear phase of the dewetting instability as the film is taken directly into the non-linear regime of it. 


\section{Acknowledgments}

This research was supported by a Marie Curie European Reintegration Grant within the $7^{\text {th }}$ European Community Framework Programme, by the European Union, Deutsche Forschungsgemeinschaft, and Universidad Politécnica de Madrid under grants PERG04-GA2008-234384, MRTN-CT-2004-005728, SFB 486 B13 and AL09-P(I+D) respectively. UT thanks MPIPKS Dresden and Peter Hänggi (Universität Augsburg) for support at various stages of the project.

* Electronic address: santiago.madruga@upm.es,smadruga@gmail.com

$\dagger$ Electronic address: u.thiele@lboro.ac.uk; URL: http://www.uwethiele.de

1 P.-G. de Gennes, "Wetting: Statics and dynamics," Rev. Mod. Phys. 57, 827-863 (1985).

2 F. Brochard-Wyart and J. Daillant, "Drying of solids wetted by thin liquid films," Can. J. Phys. 68, 1084-1088 (1989).

3 A. Oron, S. H. Davis, and S. G. Bankoff, "Long-scale evolution of thin liquid films," Rev. Mod. Phys. 69, 931-980 (1997).

4 U. Thiele, "Open questions and promising new fields in dewetting," Eur. Phys. J. E 12, 409-416 (2003).

5 R. Seemann, S. Herminghaus, C. Neto, S. Schlagowski, D. Podzimek, R. Konrad, H. Mantz, and K. Jacobs, "Dynamics and structure formation in thin polymer melt films," J. Phys.-Condes. Matter 17, S267-S290 (2005).

6 S. Kalliadasis and U. Thiele, editors, Thin Films of Soft Matter, Springer, Wien (2007), ISBN 978-3211698075.

7 G. Reiter, "Dewetting of thin polymer films," Phys. Rev. Lett. 68, 75-78 (1992).

8 A. Sharma and G. Reiter, "Instability of thin polymer films on coated substrates: Rupture, dewetting and drop formation," J. Colloid Interface Sci. 178, 383-399 (1996).

9 L. Rockford, Y. Liu, P. Mansky, T. P. Russell, M. Yoon, and S. G. J. Mochrie, "Polymers on nanoperiodic, heterogeneous surfaces," Phys. Rev. Lett. 82, 2602-2605 (1999).

10 R. Konnur, K. Kargupta, and A. Sharma, "Instability and morphology of thin liquid films on chemically heterogeneous substrates," Phys. Rev. Lett. 84, 931-934 (2000). 
11 K. Kargupta and A. Sharma, "Templating of thin films induced by dewetting on patterned surfaces," Phys. Rev. Lett. 86, 4536-4539 (2001).

12 L. Brusch, H. Kühne, U. Thiele, and M. Bär, "Dewetting of thin films on heterogeneous substrates: Pinning vs. coarsening," Phys. Rev. E 66, 011602 (2002).

13 U. Thiele, L. Brusch, M. Bestehorn, and M. Bär, "Modelling thin-film dewetting on structured substrates and templates: Bifurcation analysis and numerical simulations," Eur. Phys. J. E 11, 255-271 (2003).

14 G. Reiter, "Unstable thin polymer films: Rupture and dewetting processes," Langmuir 9, 13441351 (1993).

15 R. Xie, A. Karim, J. F. Douglas, C. C. Han, and R. A. Weiss, "Spinodal dewetting of thin polymer films," Phys. Rev. Lett. 81, 1251-1254 (1998).

16 U. Thiele, M. G. Velarde, and K. Neuffer, "Dewetting: Film rupture by nucleation in the spinodal regime," Phys. Rev. Lett. 87, 016104 (2001).

17 M. Bestehorn and K. Neuffer, "Surface patterns of laterally extended thin liquid films in three dimensions," Phys. Rev. Lett. 87, 046101 (2001).

18 K. Kargupta and A. Sharma, "Creation of ordered patterns by dewetting of thin films on homogeneous and heterogeneous substrates," J. Colloid Interface Sci. 245, 99-115 (2002).

19 O. K. C. Tsui, Y. J. Wang, H. Zhao, and B. Du, "Some views about the controversial dewetting morphology of polystyrene films," Eur. Phys. J. E 12, 417-423 (2003).

20 J. Becker, G. Grün, R. Seemann, H. Mantz, K. Jacobs, K. R. Mecke, and R. Blossey, "Complex dewetting scenarios captured by thin-film models," Nat. Mater. 2, 59-63 (2003).

21 R. Yerushalmi-Rozen, T. Kerle, and J. Klein, "Alternative dewetting pathways of thin liquid films," Science 285, 1254-1256 (1999).

22 S. Y. Heriot and R. A. L. Jones, "An interfacial instability in a transient wetting layer leads to lateral phase separation in thin spin-cast polymer-blend films," Nat. Mater. 4, 782-786 (2005).

23 R. C. Ball and R. L. Essery, "Spinodal decomposition and pattern formation near surfaces," J. Phys.: Condens. Matter 2, 10303-10320 (1990).

24 R. A. L. Jones, L. J. Norton, E. J. Kramer, F. S. Bates, and P. Wiltzius, "Surface-directed spinodal decomposition," Phys. Rev. Lett. 66, 1326-1329 (1991).

25 G. Krausch, C. A. Dai, E. J. Kramer, J. F. Marko, and F. S. Bates, "Interference of spinodal waves in thin polymer films," Macromolecules 26, 5566-5571 (1993). 
26 P. C. Hohenberg and B. I. Halperin, "Theory of dynamic critical phenomena," Rev. Mod. Phys. 49, 435-479 (1977).

27 D. M. Anderson, G. B. McFadden, and A. A. Wheeler, "Diffuse-interface methods in fluid mechanics," Ann. Rev. Fluid Mech. 30, 139-165 (1998).

28 M. E. Gurtin, D. Polignone, and J. Vinals, "Two-phase binary fluids and immiscible fluids described by an order parameter," Math. Models Meth. Appl. Sci. 6, 815-831 (1996).

29 D. Jasnow and J. Viñals, "Coarse-grained description of thermo-capillary flow," Phys. Fluids 8, 660-669 (1996).

30 N. Vladimirova, A. Malagoli, and R. Mauri, "Two-dimensional model of phase segregation in liquid binary mixtures," Phys. Rev. E 60, 6968-6977 (1999).

31 N. Vladimirova, A. Malagoli, and R. Mauri, "Diffusion-driven phase separation of deeply quenched mixtures," Phys. Rev. E 58, 7691-7699 (1998).

32 R. Borcia and M. Bestehorn, "Phase-field model for Marangoni convection in liquid-gas systems with a deformable interface," Phys. Rev. E 67, 066307 (2003).

33 R. Borcia, D. Merkt, and M. Bestehorn, "A phase-field description of surface-tension-driven instability," Int. J. Bifurcation Chaos 14, 4105-4116 (2004).

34 O. A. Frolovskaya, A. A. Nepomnyashchy, A. Oron, and A. A. Golovin, "Stability of a two-layer binary-fluid system with a diffuse interface," Phys. Fluids 20, 112105 (2008).

35 N. Clarke, "Instabilities in thin-film binary mixtures," Eur. Phys. J. E 14, 207-210 (2004).

36 N. Clarke, "Toward a model for pattern formation in ultrathin-film binary mixtures," Macromolecules 38, 6775-6778 (2005).

37 U. Thiele, S. Madruga, and L. Frastia, "Decomposition driven interface evolution for layers of binary mixtures: I. Model derivation and stratified base states," Phys. Fluids 19, 122106 (2007).

38 R. Kenzler, F. Eurich, P. Maass, B. Rinn, J. Schropp, E. Bohl, and W. Dieterich, "Phase separation in confined geometries: Solving the Cahn-Hilliard equation with generic boundary conditions," Comp. Phys. Comm. 133, 139-157 (2001).

39 E. J. Doedel, A. R. Champneys, T. F. Fairgrieve, Y. A. Kuznetsov, B. Sandstede, and X. J. Wang, AUTO97: Continuation and bifurcation software for ordinary differential equations, Concordia University, Montreal (1997).

40 E. Doedel, H. B. Keller, and J. P. Kernevez, "Numerical analysis and control of bifurcation problems (I) Bifurcation in finite dimensions," Int. J. Bifurcation Chaos 1, 493-520 (1991). 
41 E. Doedel, H. B. Keller, and J. P. Kernevez, "Numerical analysis and control of bifurcation problems (II) Bifurcation in infinite dimensions," Int. J. Bifurcation Chaos 1, 745-72 (1991).

42 U. Thiele and E. Knobloch, "Front and back instability of a liquid film on a slightly inclined plate," Phys. Fluids 15, 892-907 (2003).

43 U. Thiele, M. G. Velarde, K. Neuffer, M. Bestehorn, and Y. Pomeau, "Sliding drops in the diffuse interface model coupled to hydrodynamics," Phys. Rev. E 64, 061601 (2001).

44 U. Thiele, K. Neuffer, M. Bestehorn, Y. Pomeau, and M. G. Velarde, "Sliding drops on an inclined plane," Colloid Surf. A 206, 87-104 (2002).

45 U. Thiele and E. Knobloch, "Thin liquid films on a slightly inclined heated plate," Physica D 190, 213-248 (2004).

46 K. John, M. Bär, and U. Thiele, "Self-propelled running droplets on solid substrates driven by chemical reactions," Eur. Phys. J. E 18, 183-199 (2005).

47 U. Thiele and E. Knobloch, "On the depinning of a driven drop on a heterogeneous substrate," New J. Phys. 8, 313, 1-37 (2006).

48 U. Thiele, "Thin film evolution equations from (evaporating) dewetting liquid layers to epitaxial growth," J. Phys.-Cond. Mat. (2008), (at press).

49 B. Scheid, C. Ruyer-Quil, U. Thiele, O. A. Kabov, J. C. Legros, and P. Colinet, "Validity domain of the Benney equation including Marangoni effect for closed and open flows," J. Fluid Mech. 527, 303-335 (2005).

50 U. Thiele, J. M. Vega, and E. Knobloch, "Long-wave Marangoni instability with vibration," J. Fluid Mech. 546, 61-87 (2006).

51 U. Thiele, B. Goyeau, and M. G. Velarde, "Film flow on a porous substrate," Phys. Fluids 21, 014103 (2009).

52 E. Schäffer, Instabilities in Thin Polymer Films: Structure Formation and Pattern Transfer, Ph.D. thesis, University of Konstanz (2001).

53 M. D. Morariu, Pattern formation by capillary instabilities in thin films, Ph.D. thesis, University of Groningen (2004).

54 The parameters are $a=\left(N_{a} \rho k_{B} T / M_{w}\right)\left(-\chi / 2+N^{\prime}\right)$ and $b=\left(N_{a} \rho k_{B} T / M_{w}\right)\left(N^{\prime} / 3\right)$, with $M_{w}$ molecular weight, $N_{a}$ Avogadro number, $\rho$ density, $k_{B}$ Boltzman constant, $N^{\prime}$ number of monomers. The parameter $\chi$ is determined in Flory-Huggins theory to be $\chi(c, T)=$ $\left(P_{0}+P_{1} / T\right)\left(1+P_{2}(1+C)(1+C) / 4\right)$, where $P_{0}, P_{1}$ and $P_{2}$ are phenomenological constants. It 
is determined experimentally by Morariu and Steiner ${ }^{73}$.

55 I. Schmidt and K. Binder, "Model-calculations for wetting transitions in polymer mixtures," J Phys.-Paris 46, 1631-1644 (1985).

56 M. Geoghegan and G. Krausch, "Wetting at polymer surfaces and interfaces," Prog. Polym. Sci. 28, 261-302 (2003).

57 G. R. Strobl, The physics of polymers, Springer, Berlin (1997).

58 F. Flebbe, B. Dünweg, and K. Binder, "Phase separation versus wetting: a mean field theory for symmetrical polymer mixtures confined between selectively attractive walls," J. Phys. II France 6, 667-695 (1996).

$59 \sigma_{c}=N_{A} \rho k_{B} T s^{2} /\left[9 M_{w}(1-C)(1+C)\right]$ where $s$ is the lattice spacing.

60 D. G. Bucknall, S. Butler, and J. S. Higgins, "Real-time measurement of polymer diffusion coefficients using neutron reflection," Macromolecules 32, 5453-5456 (1999).

61 H. Qiu and M. Bousmina, "New technique allowing the quantification of diffusion at polymer polymer interfaces using rheological analysis: Theoretical and experimental results," J. Rheol. 43, 551-568 (1999).

62 R. J. Composto and E. J. Kramer, "Mutual diffusion in the miscible polymer blend polystyrene/polyxylenyl ether," Macromolecules 21, 2580-2588 (1998).

63 P. F. Green and E. J. Kramer, "Matrix effects on the diffusion of long polymer chains," Macromolecules 19, 1108-1114 (1986).

64 G. Reiter and U. Steiner, "Measurements of polymer diffusion over small distances. a check of reptation arguments," J. Phys.II 1, 659-671 (1991).

65 J. S. Langer, "An introduction to the kinetics of first-order phase transitions," in C. Godreche, editor, "Solids far from Equilibrium," pages 297-363, Cambridge University Press, Cambridge (UK). (1992).

66 R. Mauri, R. Shinnar, and G. Triantafyllou, "Spinodal decomposition in binary mixtures," Phys. Rev. E 53, 2613-2623 (1996).

67 H. P. Fischer, P. Maass, and W. Dieterich, "Diverging time and length scales of spinodal decomposition modes in thin films," Europhys. Lett. 42, 49-54 (1998).

68 M. Takashima, "Surface-tension driven instability in a horizontal liquid layer with a deformable free-surface. I. Stationary convection,” J. Phys. Soc. Jpn. 50, 2745-2750 (1981).

69 H. P. Fischer, P. Maass, and W. Dieterich, "Novel surface modes in spinodal decomposition," 
Phys. Rev. Lett. 79, 893-896 (1997).

70 A. Pototsky, M. Bestehorn, D. Merkt, and U. Thiele, "Alternative pathways of dewetting for a thin liquid two-layer film," Phys. Rev. E 70, 025201(R) (2004).

71 A. Pototsky, M. Bestehorn, D. Merkt, and U. Thiele, "Morphology changes in the evolution of liquid two-layer films," J. Chem. Phys. 122, 224711 (2005).

72 A. Pototsky, M. Bestehorn, D. Merkt, and U. Thiele, "3d surface patterns in liquid two-layer films," Europhys. Lett. 74, 665-671 (2006).

73 M. D. Morariu and U. Steiner, Personal communication (2007). 